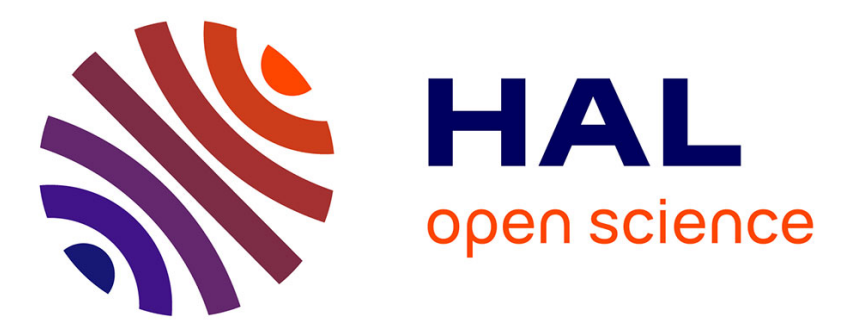

\title{
Scattering by a cylinder covered with an arbitrary distribution of impedance and application to the optimization of a tramway noise abatement system
}

Alexandre Jolibois, Denis Duhamel, Victor W. Sparrow, Jérôme Defrance, Philippe Jean

\section{To cite this version:}

Alexandre Jolibois, Denis Duhamel, Victor W. Sparrow, Jérôme Defrance, Philippe Jean. Scattering by a cylinder covered with an arbitrary distribution of impedance and application to the optimization of a tramway noise abatement system. Journal of Sound and Vibration, 2012, 331 (25), pp.5597-5622. 10.1016/J.JSV.2012.07.002 . hal-00781151

\section{HAL Id: hal-00781151 https://hal.science/hal-00781151}

Submitted on 25 Jan 2013

HAL is a multi-disciplinary open access archive for the deposit and dissemination of scientific research documents, whether they are published or not. The documents may come from teaching and research institutions in France or abroad, or from public or private research centers.
L'archive ouverte pluridisciplinaire HAL, est destinée au dépôt et à la diffusion de documents scientifiques de niveau recherche, publiés ou non, émanant des établissements d'enseignement et de recherche français ou étrangers, des laboratoires publics ou privés. 


\title{
Scattering by a cylinder covered with an arbitrary distribution of impedance and application to the optimization of a tramway noise abatement system
}

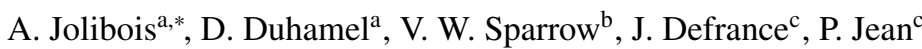 \\ ${ }^{a}$ Université Paris-Est, Laboratoire Navier, ENPC-IFSTTAR-CNRS, UMR 8205, Ecole des Ponts ParisTech, 6-8 avenue \\ Blaise Pascal, 77455 Marne-la-Vallée, France \\ ${ }^{b}$ The Pennsylvania State University, Graduate Program in Acoustics, 201 Applied Science Building, 16802 University \\ Park PA, USA \\ ${ }^{c}$ Université Paris-Est, Centre Scientifique et Technique du Bâtiment, 24 rue Joseph Fourier, 38400 \\ Saint-Martin-d'Hères, France
}

\begin{abstract}
A semi-analytical solution for the two-dimensional scattering of a line source by a cylinder with an arbitrary distribution of surface impedance and its image with respect to a vertical baffle is derived. This description is used to model the shadowing due to a low-height semi-cylindrical noise barrier close to a tramway. After validation against the boundary element method, this solution is used in a gradient-based optimization approach of the admittance distribution to maximize the broadband insertion loss in a given receiver zone. First, a hypothetical but passive distribution is found, showing an improvement of more than $20 \mathrm{~dB}(\mathrm{~A})$ with respect to a purely rigid barrier. Second, a feasible optimized surface treatment made of a porous layer and a micro-perforated resonant panel is proposed, with an improvement of $14 \mathrm{~dB}(\mathrm{~A})$ with respect to an entirely rigid barrier and $8 \mathrm{~dB}(\mathrm{~A})$ with respect to a uniform absorbent barrier. The optimization provides an automatic way of tuning the resonant panel so that the attenuation is enhanced in the frequency band where the source has the most spectral content. The benefit of using a non uniform admittance distribution is evaluated in this idealized context to be about $8 \mathrm{~dB}(\mathrm{~A})$.
\end{abstract}

Keywords: non uniform impedance, low-height barrier, cylinder scattering, impedance optimization, tramway noise

\section{Introduction}

In the past forty years, along with the increasing recognition of the influence of environmental noise on health, there has been a lot of work and effort on trying to understand how a noise barrier affects the propagation of sound and how to come up with more efficient designs $[1,2,3,4]$. It is now common to reduce the environmental noise impact of highways by flanking flat walls along tracks, and in this case the most important parameter that affects the noise reduction provided by the wall is its height: the higher the wall, the less sound is transmitted to the other side.

${ }^{*}$ Corresponding author. Tel.: + 33786386521 . Fax: + 33164153741

Email address: alexandre.jolibois@enpc.fr , auj145@psu.edu (A. Jolibois)

Preprint submitted to Journal of Sound and Vibration 
However, because building high - meaning more than two meters high - noise barriers often yields prohibitive costs since the foundation for such high and thin structures becomes incredibly large, research in environmental noise abatement has been focusing on trying to improve noise reduction performance of barriers without increasing height $[5,6]$. For instance, it has been shown that choosing a more complex shape and impedance of the top of the barrier can yield a significant improvement [3]. In particular, the use of a soft impedance has be shown to be quite efficient $[7,8,9]$, as well as optimized theoretical shapes generated by genetic algorithms [10].

However, there is more and more concern to reduce noise exposure not only close to highways but also in urban areas. However, in this case sources and receivers are separated by a few meters only, and therefore the geometrical configuration is here very different from the case of highway traffic noise mitigation where distances are much larger. Besides, implementing a wall 2 or 3 meters high along streets or tramway tracks is not a option that could be easily accepted by communities.

Hence, the possibility of using a low height protection directly between the source and the receiver started to gain interest $[11,12,13,14]$. Because the distance between source, barrier and receiver is so small, near field interference effects are expected to be stronger than in the highway case, and those effects will depend greatly on the shape and the surface boundary condition of the barrier, that is its surface admittance (the inverse of the impedance). Optimization of the impedance coverage to maximize the attenuation is therefore likely to be efficient, as shown by Thorsson [14].

However, studies on the effect of non uniform admittance distributions are still rare in the literature. Other than Thorsson's study [14], one can refer to works by Namba and Fukushige [15] and Selamet et al. [16] who showed that coupling porous materials and resonators is efficient in the context of attenuation of sound propagation in ducts. A recent work by Du et al. [17] proposed a model for studying the effect of non uniform impedance boundary conditions in rectangular cavities and showed that decay times strongly vary depending on the distribution. Therefore, there is still a need to study non-uniform admittance profiles in the context of outdoor noise abatement systems.

As an example application, it seems interesting to investigate the possibility of such a low height barrier to mitigate tramway noise. Urban tramway transportation is attractive because of its low greenhouse gas emissions, and therefore there has been a significant development of this means of transportation in main European cities (Brussels, Paris, London...). But, along with this development, the noise pollution due to the tramways has increased as well. There is therefore a need to find efficient ways to mitigate this rapidly developing noise source in urban environments, and low height noise protections could serve this purpose. A recent study [18], providing detailed measurements of sound power levels and source identification, showed that most of the noise emitted by a modern tramway comes from the rail track and the bogie (undercarriage structure) areas, which are close to the ground and therefore a low height barrier would be likely to attenuate these sources effectively. One should also point out that the noise emitted from the roof due to the HVAC (heating, ventilation and air conditioning) system mounted on certain trams, and that the low-height protection would almost not attenuate, is significant only at low rolling speeds [18]. This source of noise could be tackled for instance by implementation of barriers directly on the tram close to the HVAC, and will not be considered here. Finally, a low height protection could be implemented relatively easily very close to a tramway track since most of them already have a safety zone of several meters around them.

In summary, in this study we consider a low-height, one meter high, semi-cylindrical barrier and we allow the surface admittance to be optimized by a deterministic gradient method. Us- 
ing the semi-cylindrical geometry has several assets: aesthetically and structurally such a barrier would be integrated more easily in an urban environment, and the sound field can be calculated by semi-analytical methods even in the case of a complex admittance distribution, therefore avoiding purely numerical methods which can be extremely time-consuming. Besides, the choice of a gradient-based optimization method as opposed to an evolutionary method has been made since in this context, the gradient of the objective function can be easily obtained and therefore it seemed natural to take advantage of this information to carry on the optimization. The counterpart is that the obtained solutions will not be global optima, but such a simple approach still allows to find what key parameters influence the efficiency of the noise barrier, which is mostly what this paper is interested in.

First, we will expose the simplifying assumptions and the geometrical configuration of the problem and the semi-analytical solution for the sound field in such a configuration. Then, the optimization variables, objective function and constraints will be described. Finally, we will expose the numerical optimization results assuming two types of admittance distributions: first, as an arbitrary function depending on the position on the semi-cylinder and frequency, and second assuming a finite number of panels whose admittances are described by realistic models, such as porous layers and micro-perforated panels (MPP).

\section{Description and modeling of the barrier implementation}

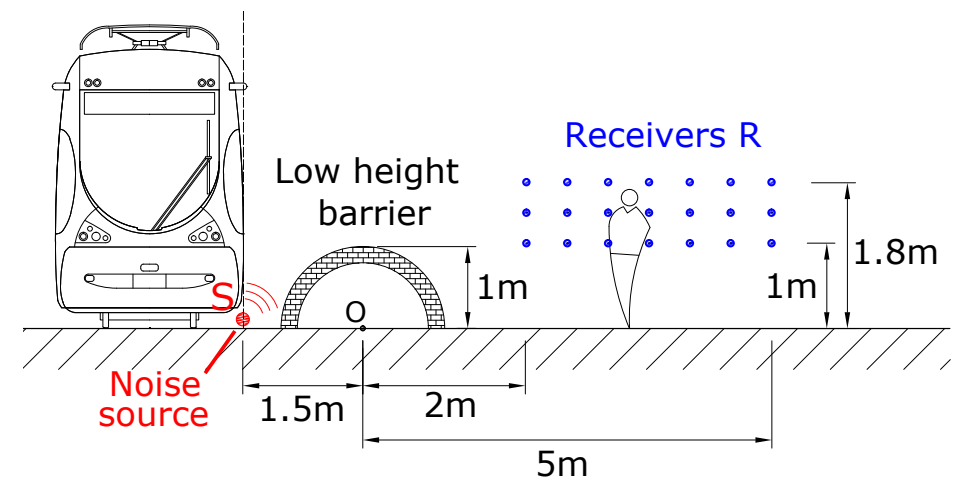

Figure 1: Geometrical configuration considered for the implementation of the tramway low-height noise barrier. The dotted line corresponds to the idealization of the tramway side as a vertical baffle.

The atmosphere is assumed to be homogeneous with a speed of sound of $c_{0}=343 \mathrm{~m} \mathrm{~s}^{-1}$ (air at $20^{\circ} \mathrm{C}$ ), which is a valid assumption since only short ranges (less than $10 \mathrm{~m}$ ) are considered here. The considered physical problem is the calculation of the acoustic pressure field $p$ close to a low-height urban noise barrier in presence of a tramway (see Fig. 1).

\subsection{Source modeling}

The noise source is modeled as an infinite, omni-directional line source on the ground. The spectral content of this source is taken to be an incoherent sum of the spectral content of the three main sources identified in [18] for a modern tram rolling at $40 \mathrm{~km} \mathrm{~h}^{-1}$ on rigid paving: rail track, powered and un-powered bogie (see Fig. 2). One can infer from the figure that most of the 
A-weighted acoustic energy is contained in the frequency range $100-5000 \mathrm{~Hz}$, which will be the range of interest in the rest of this study.

It is also assumed that the geometry is invariant along the axis of the track, which makes the problem purely two dimensional and the source a coherent line source. This assumption has been shown to be correct when predicting excess attenuations at a given frequency due to a point source and in presence of an infinitely long barrier [19], which is what we will use in the calculation of the broadband insertion loss. Besides, the 2D approximation implies that all sources contributions are "spread" uniformly along the track. Because only one coherent line source is considered, we expect strong interference effects due to the reflection on the tram, but in reality, due to the spatial incoherence of the noise sources, those interference effects are probably greatly reduced.

Also, a more realistic description of the source would probably require taking into account its directivity, as well as the incoherence along the track, which could be done for instance by following the approach described by Duhamel [19]. However, the focus of this work is to have a somewhat simple model which allows a faster evaluation of the sound field so that it can be used many times in an optimization algorithm, and therefore those effects will not be considered in this work. Furthermore, we expect that the predicted benefit of the barrier admittance to be larger to some extent than what would be observed in practice.

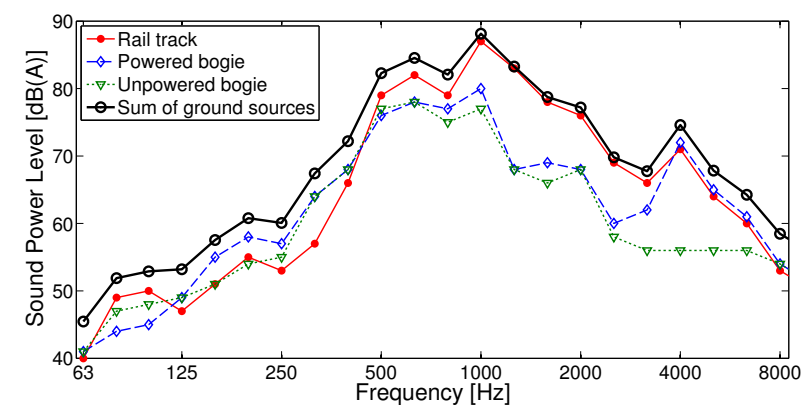

Figure 2: Comparison of third octave spectra of the different sources identified in [18] and their incoherent summation.

\subsection{Modeling of the tramway}
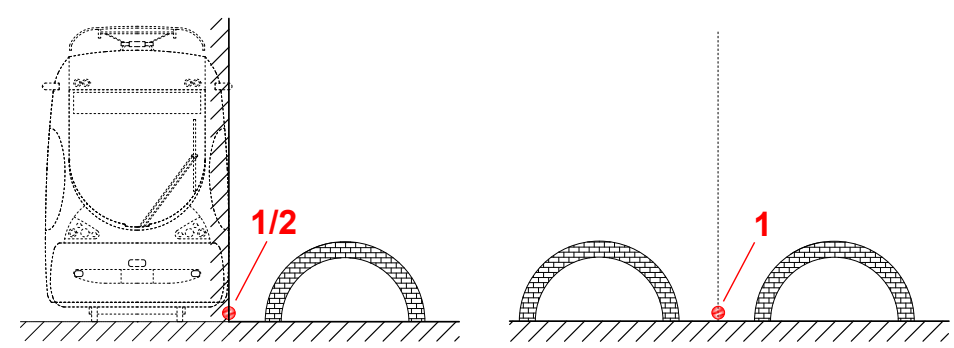

Figure 3: Schematic representing the effect of the vertical baffle. Left: original problem, in presence of the barrier, the ground and the vertical baffle. The source has an amplitude 1/2. - Right: equivalent problem with the vertical baffle implicitly taken into account. The source is scattered by the barrier and its image, and the equivalent source has now a doubled amplitude 1. 
The presence of the tramway causes the sound to bounce on its surface and to diffract at the roof edge and at the gap between the carriage and the ground. Calculation of the sound field in such an environment with a realistic cross section of the tramway could be achieved with the boundary element method (BEM). Again, since the sound field will be evaluated many times in the optimization algorithm, such a fine description of the geometry would yield an overwhelming computation time. One way to model the tramway to avoid this problem is as an infinite rigid vertical baffle placed at the location of the vertical portion of the tram. This idealization is equivalent to introducing an image barrier, symmetrical to the original one with respect to the tramway side surface (see Fig. 3). This configuration still allows semi-analytical calculation via a multi-scattering approach.

However, such a simplification ignores diffraction effects from the roof and therefore may not be accurate especially at low frequencies due to the finite height of the tramway side, and also at very high frequencies where fine details of the geometry have a significant influence on the sound field. Since those effects affect the sound field both with and without the barrier, the benefit of the barrier admittance might not depend too much on them. This assumption would require further investigation and is beyond the scope of the present study.

\subsection{Modeling of the ground}

Modeling the ground as an infinite rigid baffle is very convenient computationally because this allows application of the image theory, transforming the semi-cylindrical barrier into a whole cylinder, and allowing a semi-analytical approach. However, the finite impedance of the ground has been shown to have a large influence on the efficiency of a barrier as shown by Thorsson [14]. Measurements done by Pallas et al. [18] made on two different sites, one covered with rigid paving and the other with grass, also showed that the measured sound power was significantly reduced when the ground close to the source was covered with grass. It is possible to model the finite admittance of the ground in the BEM via a correction term in the Green's function [20], but this does not allow simple application of image theory.

Nevertheless, the ground is considered to be rigid, which would be a valid approximation in an untreated urban site covered by asphalt and concrete. This approach could also be considered as an extreme case scenario, since a softer ground like grass would probably attenuate the noise more, but may also reduce the improvement of the attenuation due to the barrier as suggested by Hutchins et al. [21]. This would also require further investigation.

\subsection{Barrier location and receiver points}

The radius of the barrier, and therefore its height, is $a=1 \mathrm{~m}$. The distance between the noise source (S) and the center of the barrier is taken to be $d=1.5 \mathrm{~m}$, which leaves a gap of $0.5 \mathrm{~m}$ between the tram and the barrier. The receiver locations (R) have been chosen to represent a range of possible locations of walking-by pedestrians ears: horizontal distance between $x_{1}=2 \mathrm{~m}$ and $x_{1}=5 \mathrm{~m}$ from the center of the barrier $(\mathrm{O})$, and height between $x_{2}=1 \mathrm{~m}$ and $x_{2}=1.8 \mathrm{~m}$ (see Fig. 1).

\subsection{Mathematical representation of the geometry}

Modeling the tramway as an infinite vertical rigid baffle is equivalent to consider a second barrier symmetrical to the first one with respect to the vertical side of the tram cross section. This implies that the admittance distribution on the image cylinder should be symmetrical to the original distribution. Besides, applying the same symmetry argument to take into account the 


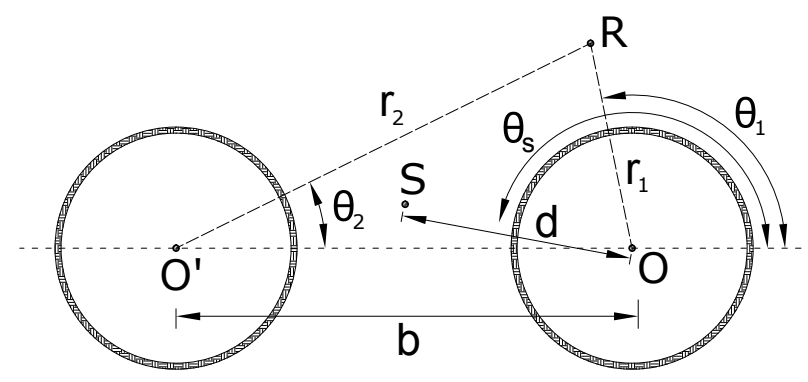

Figure 4: Geometry of the equivalent scattering problem: coordinate systems and notations.

rigid ground yields that the problem is equivalent to the scattering of a line source by two infinite cylinders covered by an arbitrary distribution of admittance (see Fig. 4). Again, this implies that on both cylinders the distribution of admittance has to be symmetrical with respect to the ground. Let us now details the notations we will use to derive the semi-analytical solution of this problem.

We will use the two cylindrical coordinate systems $\left(r_{1}, \theta_{1}\right)$ and $\left(r_{2}, \theta_{2}\right)$ respectively centered at the center of the original cylinder $(\mathrm{O})$ and that of the image cylinder $\left(\mathrm{O}^{\prime}\right)$. The reference $\theta_{1}=\theta_{2}=0$ is taken along the ground on the receiver side. The source $(\mathrm{S})$ has coordinate $\left(r_{1}=d, \theta_{1}=\theta_{s}\right)$ in the true cylinder coordinate system $\left(\theta_{s}=\pi\right.$ if the source is on the ground). The solution is derived in the frequency domain, so that the frequency $f$ is fixed and the wavenumber is $k=2 \pi f / c_{0}$. The time convention is $\mathrm{e}^{-\mathrm{i} \omega t}$.

The surface of the original cylinder is assumed to be locally reacting described by a normalized admittance function $\beta\left(\theta_{1}\right)$ with $\theta_{1} \in[0,2 \pi]$. The normalization constant is $\rho_{0} c_{0}\left(\rho_{0}\right.$ the density of air) so that $\beta$ is written in terms of the physical surface impedance $Z$ as $\beta=\rho_{0} c_{0} / Z$. The finite admittance boundary condition at the surface is written as:

$$
\left(\forall \theta_{1} \in[0,2 \pi]\right) \quad \frac{\partial p}{\partial r_{1}}\left(a, \theta_{1}\right)+\mathrm{i} k \beta\left(\theta_{1}\right) p\left(a, \theta_{1}\right)=0
$$

Because of the implicit angular periodicity and the symmetry, the distribution can be expanded as an angular Fourier series of the form:

$$
\beta\left(\theta_{1}\right)=\sum_{j=0}^{\infty} \beta_{j} \cos \left(j \theta_{1}\right)
$$

Only cosine terms are considered because $\beta$ has to be symmetric about $\theta_{1}=0$. The coefficients $\beta_{j}$ will be referred to as the admittance coefficients and uniquely define an admittance distribution. Those coefficients also depend on frequency. We will also use the notation $\boldsymbol{\beta}$ to refer to the vector containing those coefficients.

The distribution on the image cylinder $\tilde{\beta}\left(\theta_{2}\right)$ can be expanded in a similar fashion, but because of the symmetry with respect to the vertical baffle, we necessarily have:

$$
\tilde{\beta}\left(\theta_{2}\right)=\beta\left(\pi-\theta_{2}\right)=\sum_{j=0}^{\infty}(-1)^{j} \beta_{j} \cos \left(j \theta_{2}\right)
$$


The boundary condition on the image cylinder is written in a similar way:

$$
\left(\forall \theta_{2} \in[0,2 \pi]\right) \quad \frac{\partial p}{\partial r_{2}}\left(a, \theta_{2}\right)+\mathrm{i} k \tilde{\beta}\left(\theta_{2}\right) p\left(a, \theta_{2}\right)=0
$$

\section{Solution of the acoustic scattering by two non uniform impedant cylinders}

The solution described here is somewhat inspired by the solution for a constant impedant cylinder [22], and is slightly more general than the geometrical description of interest since we will not assume that the source lies on the ground. The pressure field is broken up like $p=p^{\text {in }}+p_{1}^{\mathrm{sc}}+p_{2}^{\mathrm{sc}}$ where $p^{\text {in }}$ is the incident field, $p_{1}^{\mathrm{sc}}$ is the field scattered by the true cylinder and $p_{2}^{\text {sc }}$ is the field scattered by the image cylinder.

In order to determine the incident field, let us first consider the original problem, with the semi-cylindrical barrier, the rigid ground and the vertical baffle and let the source amplitude of this problem be $1 / 2$ (see Fig. 3, left part). This amplitude is arbitrary since we are interested in calculating the attenuation, which at a given frequency does not depend on the source amplitude. The source is assumed to lie exactly on the vertical baffle (this assumption is not necessary but simply makes the calculation easier). Then, when the symmetry with respect to the vertical baffle is taken into account, the problem becomes equivalent to the scattering due to the two semi-cylinders - the original one and its image with respect to the baffle - of the field emitted by the source and its image (see Fig. 3, right part). But because the source lied exactly on the baffle, the image source is superimposed to the original one and therefore becomes equivalent to one source with a doubled amplitude, therefore an amplitude 1 . Therefore, in the equivalent problem the incident field is simply given by the 2D Green's function in presence of a rigid ground, which is given by:

$$
p^{\text {in }}=\frac{\mathrm{i}}{4}\left[H_{0}^{(1)}(k \mathrm{SR})+H_{0}^{(1)}\left(k \mathrm{~S}^{\prime} \mathrm{R}\right)\right]
$$

where (S') is the image source with respect to the ground, SR and S'R the distances between the receiver and the actual and image source respectively, and $H_{0}^{(1)}$ is the Hankel function of the first kind of order zero.

As we stated before, the presence of the ground can be taken into account by adding symmetric images of the two semi-cylindrical barriers, transforming them into whole cylinders, which allows the use of cylindrical functions to express the scattered fields. They can be represented with series of the form:

$$
p_{l}^{\mathrm{sc}}\left(r_{l}, \theta_{l}\right)=\sum_{n=0}^{\infty} \frac{a_{n}^{l}}{\epsilon_{n}} H_{n}^{(1)}\left(k r_{l}\right) \cos \left(n \theta_{l}\right)
$$

with $l=1,2, \epsilon_{n}=(2,1,1,1 \ldots)$ and $H_{n}^{(1)}$ is the Hankel functions of the first kind of order $n$. Only first order Hankel functions have been considered since the scattered fields are purely outgoing (they must satisfy the radiation condition), and only cosine terms are considered in the angular dependence since the problem is symmetrical about $\theta=0$. However, under this form it is difficult to solve for the coefficients $a_{n}^{l}$ because their magnitude sharply decrease with $n$, which is related to the fact that the matrix equation to solve would be poorly conditioned. Therefore, to partially avoid this problem, we will define new coefficients $\alpha_{n}^{l}=a_{n}^{l} H_{n}^{(1)}(k a)$ so that both scattered fields are written as:

$$
p_{l}^{\mathrm{sc}}\left(r_{l}, \theta_{l}\right)=\sum_{n=0}^{\infty} \frac{\alpha_{n}^{l}}{\epsilon_{n}} \frac{H_{n}^{(1)}\left(k r_{l}\right)}{H_{n}^{(1)}(k a)} \cos \left(n \theta_{l}\right)
$$


with $l=1,2$. Determination of the coefficients $\alpha_{n}^{l}$ comes from the boundary conditions on the original and image cylinders. This requires to express the incident field and one of the two scattered fields in the same basis of functions as the other scattered field, which basically corresponds to expand them as a Fourier series of the two spatial variables $\theta_{1}$ or $\theta_{2}$. This can be achieved using Graf's addition theorem (see [23] p.363). For instance, the contribution from the source (S) can be written as:

$$
\frac{\mathrm{i}}{4} H_{0}^{(1)}(k \mathrm{SR})=\frac{\mathrm{i}}{2} \sum_{m=0}^{\infty} \frac{1}{\epsilon_{m}} J_{m}\left(k r_{1}\right) H_{m}^{(1)}(k d) \cos \left[m\left(\theta_{1}-\theta_{s}\right)\right]
$$

where $J_{m}$ is the Bessel function of the first kind of order $m$. The field emitted by the image source (S') can be similarly written as:

$$
\frac{\mathrm{i}}{4} H_{0}^{(1)}\left(k \mathrm{~S}^{\prime} \mathrm{R}\right)=\frac{\mathrm{i}}{2} \sum_{m=0}^{\infty} \frac{1}{\epsilon_{m}} J_{m}\left(k r_{1}\right) H_{m}^{(1)}(k d) \cos \left[m\left(\theta_{1}+\theta_{s}\right)\right]
$$

so that the total incident field is given by:

$$
p^{\text {in }}\left(r_{1}, \theta_{1}\right)=\mathrm{i} \sum_{m=0}^{\infty} \frac{1}{\epsilon_{m}} J_{m}\left(k r_{1}\right) H_{m}^{(1)}(k d) \cos \left(m \theta_{s}\right) \cos \left(m \theta_{1}\right)
$$

where the fact that $\cos \left[m\left(\theta_{1}-\theta_{s}\right)\right]+\cos \left[m\left(\theta_{1}+\theta_{s}\right)\right]=2 \cos \left(m \theta_{s}\right) \cos \left(m \theta_{1}\right)$ has been used. It should also be pointed out that the expansion of the incident field emitted by (S) and (S') are valid under the condition $r_{1}<d$, which is the case at $r_{1}=a$.

The scattered field $p_{2}^{\text {sc }}$ can be expressed as a function of $r_{1}$ and $\theta_{1}$ using the expansion of the functions $H_{n}^{(1)}\left(k r_{2}\right) \cos \left(n \theta_{2}\right)$ provided by Graf's theorem:

$$
\begin{aligned}
p_{2}^{\mathrm{sc}}\left(r_{1}, \theta_{1}\right)= & \sum_{n=0}^{\infty} \frac{\alpha_{n}^{2}}{\epsilon_{n} H_{n}^{(1)}(k a)} \sum_{m=-\infty}^{\infty} H_{n+m}(k b) J_{m}\left(k r_{1}\right) \underbrace{\cos \left[m\left(\pi-\theta_{1}\right)\right]}_{=(-1)^{m} \cos \left(m \theta_{1}\right)} \\
= & \sum_{n=0}^{\infty} \frac{\alpha_{n}^{2}}{\epsilon_{n} H_{n}^{(1)}(k a)}\left[H_{n}^{(1)}(k b) J_{0}\left(k r_{1}\right)\right. \\
& \left.+\sum_{m=1}^{\infty}(-1)^{m}\left(H_{m+n}^{(1)}(k b)+(-1)^{n} H_{m-n}^{(1)}(k b)\right) J_{m}\left(k r_{1}\right) \cos \left(m \theta_{1}\right)\right]
\end{aligned}
$$

where the property $W_{-r}=(-1)^{r} W_{r}$ valid for all Bessel functions of integer order $r$ has been used. Expansions of the functions $H_{n}^{(1)}\left(k r_{2}\right) \cos \left(n \theta_{2}\right)$ are only valid where $r_{1}<b$, which is again the case at $r_{1}=a$. Similarly, we have:

$$
\begin{aligned}
p^{\mathrm{in}}\left(r_{2}, \theta_{2}\right) & =\mathrm{i} \sum_{m=0}^{\infty} \frac{1}{\epsilon_{m}} J_{m}\left(k r_{2}\right) H_{m}^{(1)}(k d) \cos \left[m\left(\pi-\theta_{s}\right)\right] \cos \left(m \theta_{2}\right) \\
p_{1}^{\mathrm{sc}}\left(r_{2}, \theta_{2}\right)= & \sum_{n=0}^{\infty} \frac{\alpha_{n}^{1}}{\epsilon_{n} H_{n}^{(1)}(k a)}\left[(-1)^{n} H_{n}^{(1)}(k b) J_{0}\left(k r_{1}\right)\right. \\
& \left.+\sum_{m=1}^{\infty}\left((-1)^{n} H_{m+n}^{(1)}(k b)+H_{m-n}^{(1)}(k b)\right) J_{m}\left(k r_{2}\right) \cos \left(m \theta_{2}\right)\right]
\end{aligned}
$$


Similarly, equation (9) is valid where $r_{2}<d$ and equation (10) where $r_{2}<b$, therefore both are valid on the surface of the image cylinder $r_{2}=a$.

Applying the finite impedance boundary condition on both cylinders and identifying Fourier coefficients for the two angular variables (see Appendix A) yields the following infinite matrix equation satisfied by the coefficients $\alpha_{n}^{1}$ and $\alpha_{n}^{2}$ :

$$
\left[\begin{array}{ll}
\mathbf{M}^{11} & \mathbf{M}^{12} \\
\mathbf{M}^{21} & \mathbf{M}^{22}
\end{array}\right]\left[\begin{array}{l}
\boldsymbol{\alpha}^{1} \\
\boldsymbol{\alpha}^{2}
\end{array}\right]=\left[\begin{array}{l}
\mathbf{e}^{1} \\
\mathbf{e}^{2}
\end{array}\right]
$$

$\mathbf{M}^{11}$ and $\mathbf{M}^{22}$ are referred to as the self-coupling matrices and $\mathbf{M}^{12}$ and $\mathbf{M}^{21}$ as the cross-coupling matrices; $\boldsymbol{\alpha}^{1}$ and $\boldsymbol{\alpha}^{2}$ are two vectors containing the coefficients $\alpha_{n}^{1}$ and $\alpha_{n}^{2}$ respectively; $\mathbf{e}^{1}$ and $\mathbf{e}^{2}$ are the two vectors containing the coefficients $e_{p}^{1}$ and $e_{p}^{2}$ corresponding to the influence of the incident field on each cylinder. Those coefficients are given by, for $p>0$ :

$$
\left\{\begin{array}{c}
e_{0}^{1}=-\frac{\mathrm{i}}{2} J_{0}^{\prime}(k a) H_{0}^{(1)}(k d)+\frac{1}{2} \sum_{j=0}^{\infty} J_{j}(k a) H_{j}^{(1)}(k d) \cos \left(j \theta_{s}\right) \beta_{j} \\
e_{p}^{1}=-\mathrm{i} J_{p}^{\prime}(k a) H_{p}^{(1)}(k d) \cos \left(p \theta_{s}\right)+\frac{1}{2} \sum_{j=0}^{\infty} \beta_{j} J_{j-p}(k a) H_{j-p}^{(1)}(k d) \cos \left[(j-p) \theta_{s}\right] \\
+\frac{1}{2} \sum_{j=0}^{\infty} \beta_{j} J_{j+p}(k a) H_{j+p}^{(1)}(k d) \cos \left[(j+p) \theta_{s}\right]
\end{array}\right.
$$

with ' is the derivative with respect to the argument. We also have for any integer value of $p$ : $e_{p}^{2}=(-1)^{p} e_{p}^{1}$.

The coefficients of the self-coupling matrix $\mathbf{M}^{11}$ are defined by, for $p, n \geqslant 0$ :

$$
\begin{gathered}
\mathbf{M}_{p n}^{11}=\frac{H_{p}^{(1)^{\prime}}(k a)}{\epsilon_{p} H_{p}^{(1)}(k a)} \delta_{p n}+\frac{\mathrm{i}}{2} \beta_{p+n}+\frac{\mathrm{i}}{2} Q_{p n} \\
\text { with } Q_{p n}= \begin{cases}0 & \text { if } n=0 \text { or } p=0 \\
\beta_{p-n} & \text { if } n<p \\
2 \beta_{0} & \text { if } n=p \\
\beta_{n-p} & \text { if } n>p\end{cases}
\end{gathered}
$$

and $\delta_{p n}$ is the Kronecker delta function. The coefficients of $\mathbf{M}^{22}$ are defined in a similar fashion replacing $\beta_{r}$ by $(-1)^{r} \beta_{r}$ for any integer value $r$. 
The coupling matrix $\mathbf{M}^{12}$ coefficients are defined by (for $n \geqslant 0, p>0$ ):

$$
\begin{aligned}
& \left(\mathbf{M}_{0 n}^{12}=\frac{1}{\epsilon_{n} H_{n}^{(1)}(k a)}\left[\left(J_{0}^{\prime}(k a)+\mathrm{i} \beta_{0} J_{0}(k a)\right) H_{n}^{(1)}(k b)\right.\right. \\
& \left.+\frac{\mathrm{i}}{2} \sum_{m=1}^{\infty}(-1)^{m} J_{m}(k a)\left(H_{n+m}^{(1)}(k b)+(-1)^{n} H_{m-n}^{(1)}(k b)\right) \beta_{m}\right] \\
& \mathbf{M}_{p n}^{12}=\frac{1}{\epsilon_{n} H_{n}^{(1)}(k a)}\left[(-1)^{p}\left(H_{n+p}^{(1)}(k b)+(-1)^{n} H_{p-n}^{(1)}(k b)\right)\left(J_{p}^{\prime}(k a)+\mathrm{i} \beta_{0} J_{p}(k a)+\frac{\mathrm{i}}{2} \beta_{2 p} J_{p}(k a)\right)\right. \\
& +\mathrm{i} \beta_{p} H_{n}^{(1)}(k b) J_{0}(k a) \\
& +\frac{\mathrm{i}}{2} \sum_{m=1}^{p-1}(-1)^{m}\left(\beta_{p-m}+\beta_{p+m}\right) J_{m}(k a)\left(H_{m+n}^{(1)}(k b)+(-1)^{n} H_{m-n}^{(1)}(k b)\right) \\
& \left.+\frac{\mathrm{i}}{2} \sum_{m=p+1}^{\infty}(-1)^{m}\left(\beta_{m-p}+\beta_{p+m}\right) J_{m}(k a)\left(H_{m+n}^{(1)}(k b)+(-1)^{n} H_{m-n}^{(1)}(k b)\right)\right]
\end{aligned}
$$

The other coupling matrix $\mathbf{M}^{21}$ is related to $\mathbf{M}^{12}$ by the relationship:

$$
(\forall p, n \geqslant 0) \quad \mathbf{M}_{p n}^{21}=(-1)^{p+n} \mathbf{M}_{p n}^{12}
$$

Equation (11) is an infinite matrix equation $\mathbf{M} \boldsymbol{\alpha}=\mathbf{e}$ with $\boldsymbol{\alpha}=\left(\boldsymbol{\alpha}^{1}, \boldsymbol{\alpha}^{2}\right)$ and $\mathbf{e}=\left(\mathbf{e}^{1}, \mathbf{e}^{2}\right)$.

\section{Numerical implementation of the solution}

\subsection{Approximation expressions for large orders}

Equation (11) requires a few approximations in order to be solved accurately. For instance, to calculate diagonal coefficients of the matrix $\mathbf{M}^{11}$ defined in equation (13), large order Bessel functions evaluations are required, which are both computationally expensive and can even lead to numerical errors. Those coefficients require the calculation of terms of the form, for a generic index $p$ and real number $w$ :

$$
\frac{H_{p}^{(1)^{\prime}}(w)}{H_{p}^{(1)}(w)}=\frac{\frac{p}{w} H_{p}^{(1)}(w)-H_{p+1}^{(1)}(w)}{H_{p}^{(1)}(w)}=\frac{p}{w}-\frac{H_{p+1}^{(1)}(w)}{H_{p}^{(1)}(w)}
$$

Therefore we need an approximation for the ratio $H_{p+1}^{(1)}(w) / H_{p}^{(1)}(w)$. Based on the asymptotic expression for large orders ([23] p.365) and numerical studies, we found that the following expression provides a approximation within less than $0.1 \%$ error for $p \geqslant \max (50,3 w)$ :

$$
\frac{H_{p+1}^{(1)}(w)}{H_{p}^{(1)}(w)} \approx \frac{2 p}{w}-\frac{w}{2 p}-\frac{w}{2 p^{2}}
$$

Right hand side vectors $\mathbf{e}_{1}$ and $\mathbf{e}_{2}$ involve infinite sums of terms containing $J_{r}(w) H_{r}^{(1)}(z)$ with $w=k a$ and $z=k d$. One can find that those terms rapidly go to zero with increasing $r$. We therefore decided to round terms of this form to zero above a certain order. We found numerically that whenever $r \geqslant 1.2 w+30$ and $z \geqslant 2 w$ (this condition is satisfied in our case), we have 
$J_{r}(w) H_{r}^{(1)}(z) \approx 0$ with a precision of $10^{-10}$. Besides, terms of the form $J_{r}^{\prime}(w) H_{r}^{(1)}(z)$ also needed in the calculation of the right hand side can be approximated in the same fashion.

Similarly, terms involved in the cross-coupling matrix $\mathbf{M}^{12}$ are of the form:

$$
c_{m n}=\frac{J_{m}(w)}{H_{n}^{(1)}(w)}\left(H_{m+n}^{(1)}(z)+(-1)^{n} H_{m-n}^{(1)}(z)\right)
$$

with $w=k a$ and $z=k b$. Those terms rapidly go to zero as well with increasing $m$ and $n$, therefore we decided to round those terms to zero as well for certain values of $m, n, w$ and $z$. Assuming $z=3 w$ (which is the case in our geometry) and defining $n_{c}(w)=1.2 w+20$, we found through numerical experiments that $\left|c_{m n}\right|<10^{-10}$ if $m \leqslant n_{c}(w)$ and $n \geqslant n_{c}(w)$, or if $m>n_{c}(w)$ for all $n$.

\subsection{Required number of terms}

All the quantities required to compute the pressure field at the receiver point have been represented until now as infinite series. Of course, to calculate numerically the pressure field at any receiver point $(\mathrm{R})$, several truncations need to be made on all these series, but without loss of accuracy.

Our first assumption lies in the representation of the admittance distribution (2): the maximal order representing the function $\beta\left(\theta_{1}\right)$ is set to $N_{\max }$, so that we have:

$$
\beta\left(\theta_{1}\right)=\sum_{j=0}^{N_{\max }} \beta_{j} \cos \left(j \theta_{1}\right)
$$

$N_{\max }$ is an indicator of how quickly the distribution varies along the barrier. This parameter is arbitrarily chosen, and is related to the number of variables in the optimization process. It will vary in the rest of this study.

Assuming that the resolution of the system is correct and therefore the $\alpha_{n}^{1}$ and $\alpha_{n}^{2}$ are accurate, we need to know how many coefficients are necessary to accurately represent the scattered field in equation (6). We call this number $N_{3}$. Numerical tests show that if the scattered fields are represented accurately at the surface of the corresponding cylinder, they will be accurate everywhere (this is related to the fact that the coefficients $\alpha_{n}^{l}$ are multiplied by the factor $H_{n}^{(1)}\left(k r_{l}\right) / H_{n}^{(1)}(k a)$ which is always less than 1 in magnitude). Assuming a rigid cylinder (for which the solution is analytical), we performed a convergence study in Matlab to ensure that the relative error in each point of the cylinder between the converged values and the truncated series are less than $10^{-6}$. It has been found that the expression of $N_{3}$ as a function of $k a$ can be approximated by a simple linear function:

$$
N_{3}=1.1 k a+10
$$

Then, we need to ensure that the incident and scattered fields expansions given in equations (7), (8), (9) and (10) are accurate. We therefore consider the generic expansion:

$$
H_{n}^{(1)}(w) \cos (n \mu)=\sum_{m=-\infty}^{\infty}(-1)^{m} H_{n+m}^{(1)}(z) J_{m}(y) \cos (m v)
$$

with the validity condition $y<z$, and the generic variables $w, \mu, y, z$ and $v$ properly defined for the expansion to be correct (see [23] p.365). From this condition, one can infer than the number of terms required to represent the function accurately depends on how close the considered point is to the validity domain boundary, which we can quantify by the ratio $\rho=y / z<1$. One can 
verify that if the series is accurate at a given $\rho$, it will be accurate as well for a lower $\rho$. We then define $Q_{n}(M, y)$ the truncated series to the $M^{\text {th }}$ order:

$$
Q_{n}(M, y)=\sum_{m=-M}^{M}(-1)^{m} H_{n+m}^{(1)}(z) J_{m}(y) \cos (m v)
$$

We call $N_{\min }(n, \rho, z)$ the number of terms needed so that the series represents the initial expanded function with a precision of $\epsilon=10^{-6}$ at ranges $y$ less than $\rho z$ :

$$
\left|H_{n}^{(1)}(x) \cos (n \theta)-Q_{n}\left(N_{\min }(n, \rho, z), \rho z\right)\right| \leqslant \epsilon\left|H_{n}^{(1)}(x) \cos (n \theta)\right|
$$

Two cases should be considered: the expansion of the incident field therefore of the function $H_{0}^{(1)}$, which is used to represent the incident field on each cylinder, and the case of the expansion of a generic function $H_{n}^{(1)}$ used in the representation of the scattered field emitted by one cylinder on the other. In the first case, the ratio $\rho$ is $a / d$, which depends on the relative closeness of the source with respect to each cylinder. In the considered geometry $\rho=2 / 3$, but we considered a more general case $\rho=0.9$. By a simple convergence study done in Matlab, consisting of adding a term to the expansion until the condition (16) is met, the values of $N_{\min }(0,0.9, z)$ can be found as a function of $z$. The range $z \in[10,200]$ has been considered in the numerical tests. It has been found that this expression can be approximately fitted by the piecewise linear function:

$$
N_{\min }(0,0.9, z)= \begin{cases}0.61 z+100 & \text { if } z \leqslant 200 \\ 0.91 z+40 & \text { elsewhere }\end{cases}
$$

In the second case, the value of $\rho$ is $a / b$ with $b$ the distance between the two cylinders centers. By construction of the image cylinder, $\rho$ here is always less than $1 / 2$. However, in order to be as general as possible, it has been decided to consider the limiting case $\rho=1 / 2$ to derive the required number of terms, even though in our case $\rho=1 / 3$. It should pointed out in this case that for values of $x$ less than $n$, the function $H_{n}^{(1)}(x)$ is dominated by the divergent part of the Neumann function and therefore ensuring the strict validity of the condition (16) is very difficult to achieve since for low values of $x$, the field increases very rapidly as a function of $n$ and decreases very rapidly as a function of $x$. We therefore considered as a stop criterion the fact that the condition (16) has to be true where the field $H_{n}^{(1)}(x)$ is larger in value. Similar numerical calculations in Matlab then yield the values of $N_{\min }(n, 0.5, z)$. The considered ranges are $z \in[10,200]$ and $n \in[10,100]$. A conservative simplified expression for $N_{\min }(n, 0.5, z)$ is then derived from simple fitting:

$$
N_{\min }(n, 0.5, z)= \begin{cases}1.39 n+41 & \text { if } z \leqslant 2.48 n+47 \\ 0.56 z+15 & \text { elsewhere }\end{cases}
$$

Now, let $N_{1}$ the considered number of terms in the series in equations (7), (8), (9) and (10). Because of the accuracy requirement on the incident field and the scattered field series expansions, $N_{1}$ can be defined as:

$$
N_{1}=\max \left(N_{\min }(0,0.9, k d), N_{\min }\left(N_{3}, 0.5, k b\right)\right)
$$

In our case $(d=1.5 \mathrm{~m}, b=2 d, a=1 \mathrm{~m}$ and frequency range [100,5000] $\mathrm{Hz})$, the expression for $N_{1}$ as a function of $k a$ simplifies to:

$$
N_{1}=\max (1.53 k a+55,0.92 k a+100)
$$


Finally, we call $N_{2}$ the size of the vectors $\boldsymbol{\alpha}^{1}$ and $\boldsymbol{\alpha}^{2}$ that we solve for, which is half the size of the matrix M. This parameter must be chosen so that the calculation of the coefficients $\alpha_{n}^{l}$ is accurate. This number is not easy to estimate since it might a priori depend on several other parameters: normalized wavenumbers $k a, k b$ and $k d$, order $N_{\max }$, but also on the values $\beta_{j}$ themselves. In order to derive a simple general expression for $N_{2}$, we will assume a set of admittance coefficients of the form $\beta_{j}=(1+\mathrm{i}) /(j+1)$ which represents a typical decrease of Fourier coefficients. Besides, we will assume $d=1.5 a$ and $b=2 d$ (which is the case for the chosen geometrical configuration).

The convergence tests have been carried on as follows. For different values of frequencies (between 100 and $5000 \mathrm{~Hz}$ ) and $N_{\max }$ (between 10 and 100), a reference "converged" vector $\boldsymbol{\alpha}_{\text {ref }}$ is calculated assuming a fixed large $N_{2}$ (typically 400). Then, we progressively increase the size of the two vectors $\alpha_{1}$ and $\alpha_{2}$ until the relative error of all $N_{3}$ first coefficients with respect to the reference values is less than $10^{-6}$. This yields a set of numbers $N_{2}$ which depends on $N_{\max }$ and frequency. From this dataset and simple fitting, we found that the following simple linear function is a conservative estimate of $N_{2}$ :

$$
N_{2}=1.1 k a+N_{\max }+30
$$

The number of considered coefficients $N$ also has to meet the accuracy requirement on the Hankel functions expansions and of the scattered field, and therefore should be greater than $N_{1}$ and $N_{3}$ :

$$
N=\max \left(N_{1}, N_{2}, N_{3}\right)
$$

with the three parameters $N_{1}, N_{2}$ and $N_{3}$ given by equations (17), (18) and (15). Using the truncation parameter $N$, one can invert the system (11), compute the two scattered fields using equations (6) with $N_{3}$ terms, and finally compute the total pressure field by adding the incident field (4). This approach is therefore semi-analytical since the calculation of the inverse is not explicit. However, assembling the system (11) is fast and therefore the calculation of the pressure field is efficient, even on a broad range of frequency. This is why this method can be easily coupled with an optimization algorithm and therefore used to find better distributions of admittance on the semi-cylindrical low-height barrier.

\subsection{Validation of the solution}

The calculation of the pressure field around the semi-cylindrical barrier can also be achieved numerically using the BEM. We used the 2D BEM software MICADO developed at the CSTB by Jean [24] to validate the semi-analytical scattering solution detailed in the previous section. As an example, the barrier is here covered with two porous layers modeled with the Delany \& Bazley model [25]. The calculation of the attenuation (or insertion loss) in $\mathrm{dB}$ is performed at all frequencies from 80 to $5000 \mathrm{~Hz}$, with a step of $20 \mathrm{~Hz}$, at the receiver point of Cartesian coordinates $\left(x_{1}=7 \mathrm{~m}, x_{2}=1.8 \mathrm{~m}\right)$ from the origin $(\mathrm{O})$, center of the original cylinder. Results are presented in Fig. 5. With a requirement of 6 elements per wavelength, on a standard desktop computer, MICADO takes about $700 \mathrm{~s}$ to do this calculation whereas the scattering solution implemented in Matlab takes about $100 \mathrm{~s}$. Agreement is excellent except at very high frequencies where a difference of a few $\mathrm{dBs}$ is observed.

\section{Choice of the objective function}

We are now able to calculate the complex pressure amplitude $p(R, f)$ at each frequency $f$ and at each receiver point $(\mathrm{R})$, for a unit source amplitude. Since the $2 \mathrm{D}$ approximation is only valid 


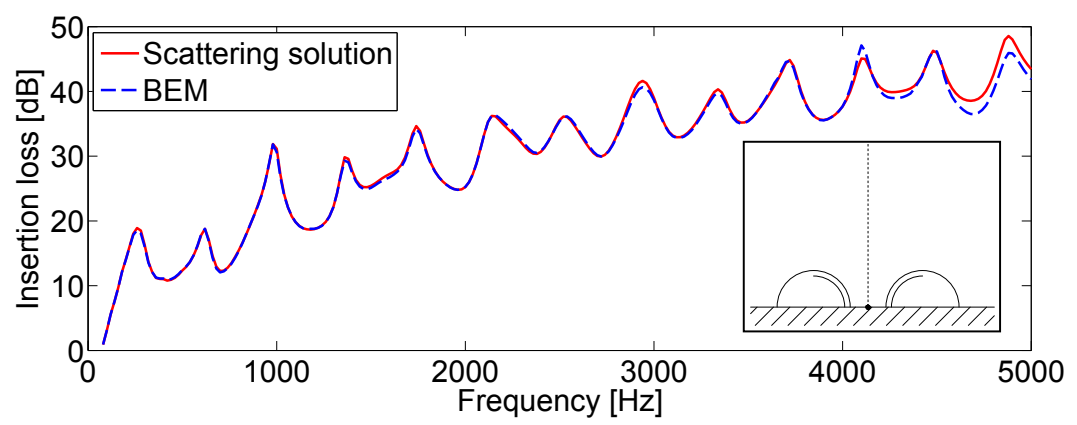

Figure 5: Attenuation in $\mathrm{dB}$ at the receiver point of Cartesian coordinates $\left(x_{1}=7 \mathrm{~m}, x_{2}=1.8 \mathrm{~m}\right)$ from the origin $(\mathrm{O})$ in presence of the true cylinder and its image calculated with the scattering solution (solid line) and the BEM (dashed line). The source location $(\mathrm{S})$ in the same Cartesian coordinates system is $\left(x_{1}=-1.5 \mathrm{~m}, x_{2}=0 \mathrm{~m}\right)$. The barrier is covered with two Delany-Bazley layers of depth $d=10 \mathrm{~cm}$ with flow resistivities $\sigma=30000 \mathrm{kPa} \mathrm{s} \mathrm{m}^{-2}$ on the receiver side (single line on the schematic) and $\sigma=30 \mathrm{kPa} \mathrm{s} \mathrm{m}^{-2}$ on the source side (double line on the schematic).

to calculate attenuations in narrow frequency bands, one possible objective function to minimize is a weighted attenuation taking into account the frequency content of the source $[5,6]$.

\subsection{Attenuation and broadband insertion loss}

From the power levels measured by Pallas et al. [18] and shown in Fig. 2, one can define an equivalent A-weighted pressure "amplitude" $S$ for each third-octave band between 100 and $5000 \mathrm{~Hz}$ by converting the power level $L_{w}$ in $\mathrm{dB}$ shown in Fig. 2 to an amplitude-like quantity: $S=10^{L_{w} / 10}$. However, one usually needs to accurately estimate the attenuation in a given band by averaging the pressure field over several frequencies. In order to limit the computation time of the objective function, we decided to consider only sixth-octave frequencies, referred to as $f_{q}$, and assign to each of them a linearly interpolated amplitude $S_{q}$, based on the power spectrum. In the range $100-5000 \mathrm{~Hz}$, this yields 35 frequencies. The average attenuation at each sixth-octave frequency calculated over all receiver points $R_{\mathrm{i}}$ defined in section 2.4 is then:

$$
A_{q}=\frac{\sum_{i}\left|p\left(R_{\mathrm{i}}, f_{q}\right)\right|^{2}}{\sum_{i}\left|p^{\mathrm{in}}\left(R_{i}, f_{q}\right)\right|^{2}}
$$

and finally the broadband weighted attenuation is:

$$
g=\frac{\sum_{q} S_{q} A_{q}}{\sum_{q} S_{q}}
$$

This is the function we would like to minimize, which only depends on the admittance distribution and therefore on the coefficients $\beta_{j}$ defined in section 2.5. One can also calculate from the objective function a broadband insertion loss in $\mathrm{dB}(\mathrm{A})$ defined by $\mathrm{IL}=-10 \log g$.

There is one major drawback of the approach we just exposed. Because the broadband objective function is an average over a set of frequencies, and is therefore independent of frequency, this implicitly assumes that the admittance distribution, which is our optimization variable via the coefficients $\beta_{j}$, is assumed constant over frequency as well. This assumption is unfortunately not realistic at all since all materials have a frequency dependent admittance. One way to overcome this problem is to perform an optimization at each frequency, taking as objective function 
to minimize the average attenuation at each frequency $A\left(f_{q}\right)$. The counterpart of this approach is that the frequency content of the source does not influence the result any more, but, since the admittance is optimized at all frequencies, the resulting admittance distribution will probably be efficient for any kind of source spectral content.

However, if we constrain the admittance distribution on the semi-cylinder more, for instance by assuming that it is due to a finite number of panels with a frequency dependent admittance described by a few parameters, we can use the weighted broadband attenuation and use the chain rule to calculate the gradient with respect to the parameters as a function of the gradient with respect to the admittance coefficients $\beta_{j}$. This approach will be used in section 7 .

The choice to optimize the sound transmitted at a few discrete frequencies, the sixth-octave frequencies, was made to keep the evaluation of the objective function somewhat reasonable time-wise. However, we have to check that the solution of the optimization process does not provide good attenuation only at those discrete frequencies and not so much at all the other intermediate frequencies. Therefore, after each run of the optimization algorithm we will also compute the attenuation on a finer mesh of frequencies, typically 20 frequencies per third-octave band. This will allow accurate calculation of the source-dependent broadband as well as thirdoctave insertion losses.

\subsection{Gradient of the attenuation}

Most deterministic optimization algorithms are based on the gradient of the objective function. It could be computed numerically by finite difference, but in this context it is possible to come up with a semi-analytical calculation of the gradient in order to have more accurate and faster results.

Both considered objective functions are directly related to the attenuations $A_{q}$ at a given frequency, therefore we only need to calculate its gradient with respect to the admittance coefficients $\partial A_{q} / \partial \beta_{j}$. Then, the gradient of the broadband attenuation is:

$$
\frac{\partial g}{\partial \beta_{j}}=\frac{1}{\sum_{q} S_{q}} \sum_{q} S_{q} \frac{\partial A_{q}}{\partial \beta_{j}}
$$

Since the incident field does not depend on the barrier, we have for any index $j \in\left[0, N_{\max }\right]$ :

$$
\begin{aligned}
\frac{\partial A_{q}}{\partial \beta_{j}}= & \frac{1}{\sum_{i}\left|p^{\text {in }}\left(R_{i}, f_{q}\right)\right|^{2}} \sum_{i} \frac{\partial\left(\left|p\left(R_{i}, f_{q}\right)\right|^{2}\right)}{\partial \beta_{j}} \\
& =\frac{2}{\sum_{i}\left|p^{\text {in }}\left(R_{i}, f_{q}\right)\right|^{2}} \sum_{\mathrm{i}} \operatorname{Re}\left[p\left(R_{i}, f_{q}\right)^{*}\left(\frac{\partial p_{1}^{\mathrm{sc}}\left(R_{i}, f_{q}\right)}{\partial \beta_{j}}+\frac{\partial p_{2}^{\mathrm{sc}}\left(R_{i}, f_{q}\right)}{\partial \beta_{j}}\right)\right]
\end{aligned}
$$

with * is the complex conjugation. We are left with the calculation of the gradient of the scattered fields with respect to the admittance coefficients. Using the definition (6), we have:

$$
\frac{\partial p_{l}^{\mathrm{sc}}}{\partial \beta_{j}}=\sum_{n=0}^{\infty} \frac{1}{\epsilon_{n}} \frac{\partial \alpha_{n}^{l}}{\partial \beta_{j}} \frac{H_{n}^{(1)}\left(k r_{l}\right)}{H_{n}^{(1)}(k a)} \cos \left(n \theta_{l}\right)
$$

with $l=1,2$. The calculation of the gradient of the attenuation is therefore based on the gradient of the vector-valued function $\alpha(\beta)$. By definition of $\alpha$, we have:

$$
\mathbf{M}(\beta) \boldsymbol{\alpha}(\beta)=\mathbf{e}(\beta)
$$


Taking the derivative with respect to $\beta_{j}$ and rearranging, we end up with:

$$
\mathbf{M} \frac{\partial \boldsymbol{\alpha}}{\partial \beta_{j}}=\frac{\partial \mathbf{e}}{\partial \beta_{j}}-\frac{\partial \mathbf{M}}{\partial \beta_{j}} \boldsymbol{\alpha}
$$

From equations (12), (13) and (14), one can obtain the terms $\partial \mathbf{e} / \partial \beta_{j}$ and $\partial \mathbf{M} / \partial \beta_{j}$. Then, if $\alpha$ is known as well, equation (20) is another system involving the matrix $\mathbf{M}$, which can be solved numerically to obtain the partial derivatives $\partial \alpha / \partial \beta_{j}$.

However, the admittance coefficients have been implicitly assumed real in this analysis. To calculate the gradient with respect to the imaginary part $\operatorname{Im} \beta_{j}$, one can use the previous approach and use the fact that the following relationship holds:

$$
\frac{\partial \boldsymbol{\alpha}}{\partial\left(\operatorname{Im} \beta_{j}\right)}=\mathrm{i} \frac{\partial \boldsymbol{\alpha}}{\partial\left(\operatorname{Re} \beta_{j}\right)}
$$

Therefore, one can compute the first $N_{\max }+1$ components of the gradients of $\alpha$ corresponding to the derivative with respect to $\operatorname{Re} \beta_{0}, \operatorname{Re} \beta_{1}, \ldots, \operatorname{Re} \beta_{N_{\max }}$ by solving equation (20), and then using the relationship (21) to compute the remaining components of the gradient. Once the total gradient is known, one can compute the derivative of $p_{1}^{\mathrm{sc}}$ and $p_{2}^{\mathrm{sc}}$, and finally that of the attenuation by using the correct component of the gradient.

\section{First optimization case: ideal admittance distribution}

The optimization is here performed at each frequency $f_{q}$ and the result is a set of $N_{\max }+1$ admittance coefficients $\beta_{j q}^{*}$ at each frequency which can take any complex values (under some constraints that we specify below). We will also consider several values of $N_{\max }$ to measure how this parameter influence the gain obtained in the noise reduction.

\subsection{Energy absorption constraint}

However, even if we are here interested in an arbitrary distribution of admittance, we would like to ensure that the hypothetical distribution of materials covering the barrier is somewhat physical, especially the material can only absorb acoustic energy and cannot provide more energy. In this sense the barrier is a passive noise control device. Mathematically, this is ensured if the real part of the admittance is non-negative at any point of the barrier, namely:

$$
\left(\forall \theta_{1} \in[0,2 \pi]\right) \quad \operatorname{Re}\left[\beta\left(\theta_{1}\right)\right]=\sum_{j=0}^{N_{\max }} \operatorname{Re}\left(\beta_{j}\right) \cos \left(j \theta_{1}\right) \geqslant 0
$$

Under this form, the constraint would not be easily implemented since the angle varies continuously. Reporting this constraint on the coefficients $\beta_{j}$ only can be done based on the following observation:

$$
\begin{aligned}
\sum_{j=0}^{N_{\max }} \operatorname{Re}\left(\beta_{j}\right) \cos \left(j \theta_{1}\right) & =\operatorname{Re}\left(\beta_{0}\right)+\sum_{j=1}^{N_{\max }} \underbrace{\operatorname{sgn}\left[\operatorname{Re}\left(\beta_{j}\right)\right] \operatorname{Re}\left(\beta_{j}\right)}_{\geqslant 0} \underbrace{\operatorname{sgn}\left[\operatorname{Re}\left(\beta_{j}\right)\right] \cos \left(j \theta_{1}\right)}_{\geqslant-1} \\
& \geqslant \operatorname{Re}\left(\beta_{0}\right)-\sum_{j=1}^{N_{\max }} \operatorname{sgn}\left[\operatorname{Re}\left(\beta_{j}\right)\right] \operatorname{Re}\left(\beta_{j}\right)
\end{aligned}
$$


Therefore we have:

$$
\begin{array}{ll} 
& \operatorname{Re}\left(\beta_{0}\right) \pm \operatorname{Re}\left(\beta_{1}\right) \pm \operatorname{Re}\left(\beta_{2}\right) \pm \ldots \pm \operatorname{Re}\left(\beta_{N_{\max }}\right) \geqslant 0 \\
\Rightarrow \quad & \operatorname{Re}\left[\beta\left(\theta_{1}\right)\right] \geqslant 0 \quad\left(\forall \theta_{1} \in[0,2 \pi]\right)
\end{array}
$$

Using this property, the energy absorption constraint can be implemented as a set of $2^{N_{\max }}$ linear constraints, corresponding to all the combinations of pluses and minuses in equation (22).

\subsection{Bounds on the admittance coefficients values}

Even if in this first case, we are looking for the "ideal" distribution which may or may not be physically feasible, we will restrain the value of the admittance to somewhat reasonable values. Let us recall that the case of a perfectly rigid surface corresponds to $\beta=0$. The case $\beta=1$ means that the impedance of the surface matches the impedance of air, which could be considered as the "perfect absorption" condition. Finally, the case $\beta=\infty$ means that the surface is acoustically soft, which would be the case for a sound wave propagating in air impinging on a much lighter and less dense gas, or in some resonant conditions. According to Möser and Volz [7], a soft impedance on a barrier cylindrical top is very effective, but is difficult to achieve in practice.

We will then consider that both the real and imaginary part of $\beta$ should approximately be in the range $[-1,1]$. Ideally, this bound should be applied to both the real and imaginary part of $\beta$ at each angle $\theta$, which could be achieved by using the same kind of approach that we used to ensure the positive real part. However, in order to limit the number of constraints, we decided to use a less restrictive but simpler criterion on the coefficients by bounding each of them directly in the range $[-1 / 2,1 / 2]$. This type of constraint makes it possible for the admittance $\beta(\theta)$ to be much higher than 1 at some angles, but this somewhat simplistic approach has the main advantage that when $N_{\max }$ increases, the search space becomes increasingly large and contains all the possible configurations for lower $N_{\max }$. This will allow us to perform a sort of convergence study on the effect of $N_{\max }$.

\subsection{Implementation details}

As explained in the energy absorption constrain section, the number of constraints necessary to ensure the positivity of the real part of the admittance is $2^{N_{\max }}$, which increases very rapidly with $N_{\max }$. To limit the size of the matrices, the analysis has been limited to values of $N_{\max }$ less than 10 .

The attenuation $A_{q}$ at each frequency $f_{q}$ is nonlinear in the vector of real and imaginary parts of admittance coefficients, but the energy absorption constraint can be expressed in a linear form as stated above. Besides, each coefficient is bounded between $-1 / 2$ and $+1 / 2$. The minimization is therefore a nonlinear optimization problem with linear inequality constraints.

The optimization algorithm is the interior-point algorithm, which consists in a sequence of nonlinear equality constrained minimizations of a perturbed function equal to the objective function plus a logarithmic barrier term related to the inequality constraints and depending on a decreasing parameter (details on this algorithm can for instance be found in [26]). The strict feasibility of the solution at each iteration is usually ensured with this approach. This algorithm is run at all sixth-octave frequencies between 100 and $5000 \mathrm{~Hz}$. Besides, for each $N_{\max }$, the solution of the whole optimization process is a set of admittance coefficients $\beta_{j q}^{*}$ at each sixth-octave frequency $f_{q}$. The starting point for the first frequency is $\left(\beta_{0}, \beta_{1}, \ldots\right)=(0,0, \ldots)$, and once the optimal solution is found at a frequency $f_{q}$, the solution $\beta_{j q}^{*}$ is used as starting point for the next frequency $f_{q+1}$. This way we expect a somewhat continuous variation of the admittance with 
frequency, which is what would be observed physically on actual materials, and also a faster convergence. However, this approach is also more likely to "lock-in" the distribution in a particular local minimum. Then, the broadband efficiency IL in $\mathrm{dB}(\mathrm{A})$, based on the finer frequency mesh (20 frequencies per third-octave band) as well as the source spectral content, is computed by interpolating the admittance distribution between sixth-octave frequencies.

Finally, one should recall that this type of algorithm can only converge to a local solution at each frequency, which implies there is no guarantee that the solutions are actual global optima. In particular, even though the search space increases with increasing $N_{\max }$, we cannot expect the insertion loss at each frequency to increase with increasing $N_{\max }$, whereas it should be the case if the global optimum was found.

\subsection{Results}

\begin{tabular}{|c|c|c|c|c|c|c|c|c|c|}
\hline \multirow[b]{2}{*}{ Distributions } & \multirow[b]{2}{*}{ Rigid } & \multirow[b]{2}{*}{ Soft } & \multicolumn{7}{|c|}{ Optimized distributions obtained at different $N_{\max }$} \\
\hline & & & 0 & 1 & 2 & 4 & 6 & 8 & 10 \\
\hline Broadl & 8.7 & 23.1 & 21.6 & 29.8 & 30.6 & 30.5 & 31.4 & 32.8 & 33.5 \\
\hline
\end{tabular}

Table 1: Comparison of the broadband insertion losses in $\mathrm{dB}(\mathrm{A})$ obtained at the solutions of the optimization process as of function of $N_{\max }$. Values of the insertion loss for a purely rigid and purely soft cylinder are also shown for reference.

First, we compared the values of the broadband efficiency using different values of $N_{\max }$ (see Table 1). We also added the values obtained for a entirely rigid barrier $(\beta=0)$ and a perfectly soft barrier $(\beta=\infty)$ as references. The first comment we can make is that the optimization process yields a very significant improvement between 10 and $20 \mathrm{~dB}$ (A) upon a purely rigid barrier, in all cases. Besides, efficiencies of the obtained solutions can be higher than the perfectly soft case (by about $10 \mathrm{~dB}(\mathrm{~A})$ for $N_{\max }=10$ ).

Also, one can notice than the biggest increase in efficiency - $8 \mathrm{~dB}(\mathrm{~A})$ - happens between $N_{\max }=0$ (uniform admittance) and $N_{\max }=1$ (non uniform). This suggests that there is a significant benefit of covering the semi-cylinder with a non uniform distribution of admittance, as opposed to cover only one kind of surface treatment. Besides, the general trend of the efficiency is increasing with $N_{\max }$, but grows slower and slower.

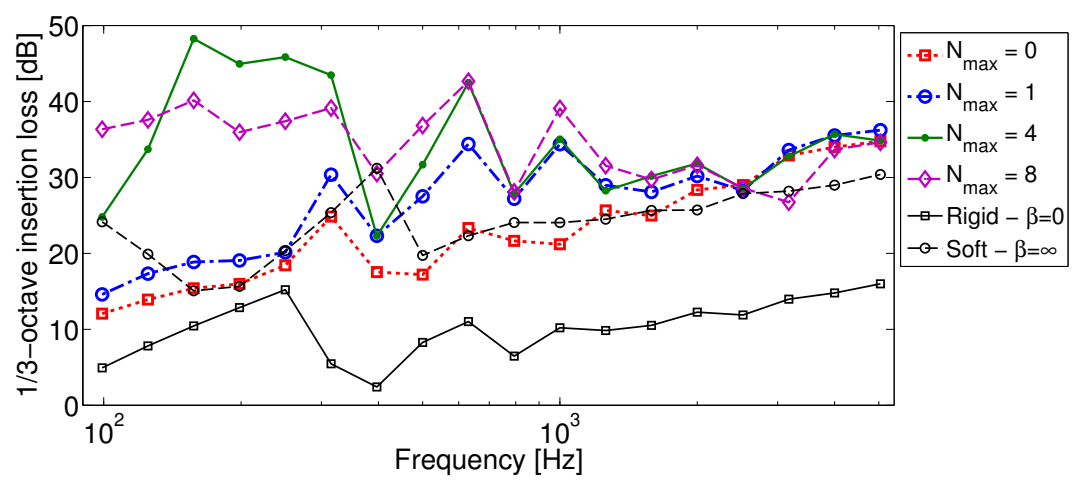

Figure 6: Comparison of predicted third-octave insertion losses in $\mathrm{dB}$ for several solutions obtained at different $N_{\text {max }}$ $(0,1,2,4$ and 8$)$ as well as for rigid and soft coverages. 
In order to compare the frequency dependence of the different distributions efficiencies, thirdoctave insertion losses are presented (see Fig. 6). One can notice that above $1000 \mathrm{~Hz}$, most solutions behave in a somewhat similar manner. However, the attenuation at low frequencies seem to strongly depend on $N_{\max }$, that is how quickly the impedance can vary along the cylinder.

An important comment should also be made about the fact that the solutions we came up with are unlikely to be global optima. From Fig. 6, one can for instance see that the insertion loss between 150 and $300 \mathrm{~Hz}$ is actually higher in the solution at $N_{\max }=4$ than for the solution at $N_{\max }=8$, which should not be the case if the solutions were truly global since the search space increases with $N_{\max }$. This is also shown in the broadband insertion losses which are not strictly monotonic with $N_{\max }$. However, obtained attenuations are still very high and show a very significant improvement, even though they do not correspond to global optima.

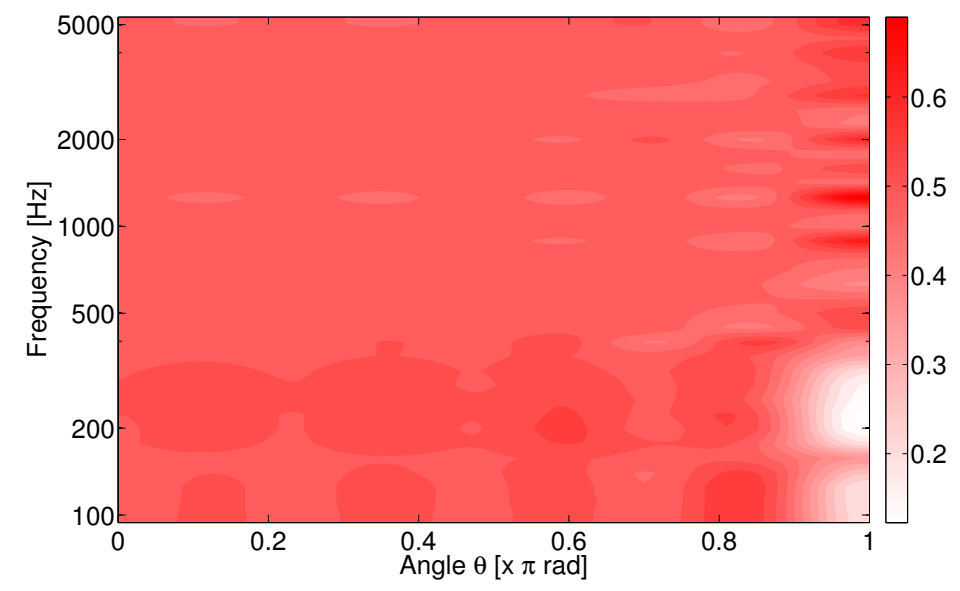

Figure 7: Real part of the admittance distribution $\operatorname{Re} \beta$ as a function of angle and frequency for the optimized solution obtained at $N_{\max }=8$.

One can also look more closely at the generated admittance distributions, as a function of frequency and angle, for instance in the case $N_{\max }=8$ (see Fig. 7 and 8). The obtained admittance real part is fairly constant (of value $1 / 2$ ), but for the imaginary part, one can notice that there is little variation of the admittance on the receiver side $\left(\theta_{1}<\pi / 2\right)$, but the variation is much stronger on the source side $\left(\theta_{1}>\pi / 2\right)$. This seems reasonable since the field is stronger close to the source and therefore it is likely that most of the interaction between the incident field and the barrier happens on this side. Besides, one should point out that the value of admittance are somewhat realistic at least in terms of magnitude since both the real and imaginary part are less than 2 on most of the semi-cylinder and at most frequencies.

On the source side, the imaginary part also undergoes strong variation with frequency and position, changing from positive to negative, suggesting that destructive interference effects related to the phase of the admittance alter the attenuation significantly in the receiver zone. Good attenuation therefore seems not only to be related to energy absorption at the surface of the barrier (real part of the admittance) but also on interference effects between the direct and scattered fields. However, if only the imaginary part of the admittance is present (again assuming the solution obtained at $N_{\max }=8$ ), the efficiency drops to $7.3 \mathrm{~dB}(\mathrm{~A})$, whereas if only the real part of the admittance is present the efficiency is $21.4 \mathrm{~dB}(\mathrm{~A})$, both lower than that of the obtained solution, 


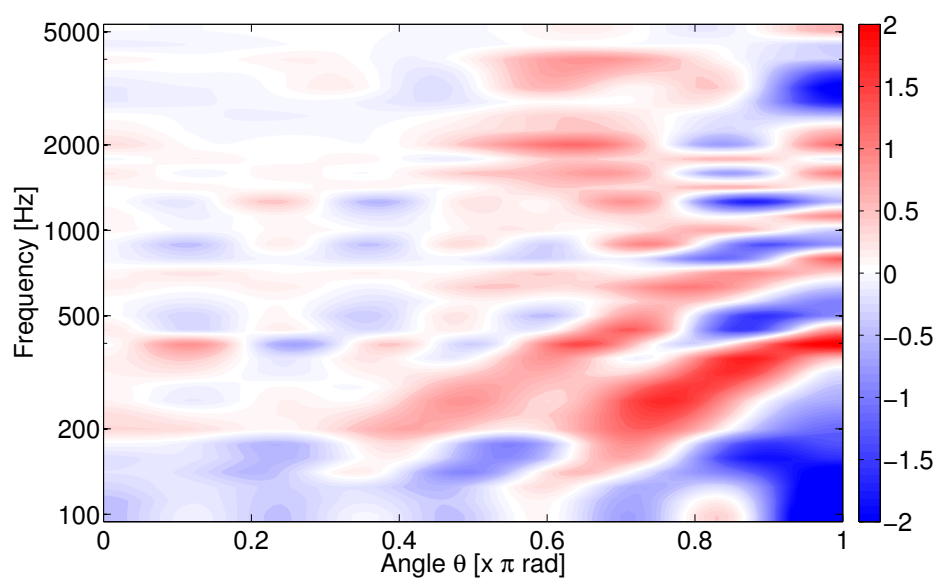

Figure 8: Imaginary part of the admittance distribution $\operatorname{Im} \beta$ as a function of angle and frequency for the optimized solution obtained at $N_{\max }=8$.

which suggests that the two effects (energy absorption and phase-related interference effects) can both be important for the attenuation due to the barrier.

Finally one could point out that interference effects are strongly related to the coherent nature of the line source and would be probably be greatly reduced if the incoherence of the source was taken into account.

\subsection{Conclusion}

This first study, somewhat theoretical in essence, helped us identify several important features in how the admittance distribution influences the noise reduction at the receivers. First, all obtained solutions have a much better performance than the purely rigid case and also better than the purely soft case. This suggests that very significant improvement can be achieved due to the admittance distribution of the barrier. Second, considering a non uniform distribution, therefore a non zero $N_{\max }$, can yield a very significant improvement of at least $8 \mathrm{~dB}(\mathrm{~A})$ in the efficiency in comparison to the optimal efficiency obtained for a uniform admittance. Treating the surface of the barrier with different materials can therefore improve its efficiency. Besides, aside from the absorption effect related to the real part of the admittance, it seems that the variation of the imaginary part of the admittance, with frequency and with position, can also play a part in the attenuation due to the barrier, related to interference effects. Therefore, the use of resonator-like treatments coupled with more traditional absorbent treatments may be efficient in this context, which seems consistent with the findings of Möser and Volz [7]. We will investigate a more physical example of this type of distribution in the following section.

\section{Second optimization case: finite number of panels with realistic impedance models}

In this section, we will constrain the problem more by assuming that the barrier is covered by a finite number of panels (see Fig. 9). Each of those panels (indexed by $P \in\left[1: N_{p}\right]$ ) lies between the two angles $\varphi_{P-1}$ and $\varphi_{P}$ (with the convention $\varphi_{0}=0$ and $\varphi_{N_{p}}=\pi$ ). The angles $\varphi_{1}$ to $\varphi_{N_{p}-1}$ are design variables subject to the constraint $0=\varphi_{0} \leqslant \varphi_{1} \leqslant \varphi_{2} \leqslant \ldots \leqslant \varphi_{N_{p}}=\pi$. 


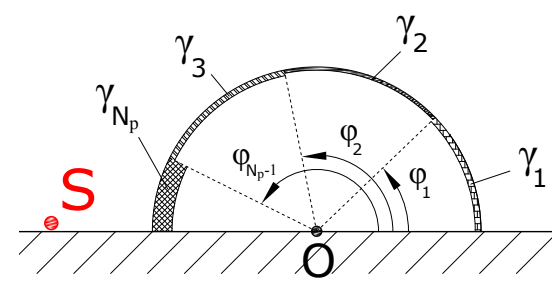

Figure 9: Cylindrical barrier covered with a finite number of panels $N_{p}$. Each panel indexed by $P \in\left[1: N_{p}\right]$ has an admittance $\gamma_{P}(f)$ described by a realistic model and lies between the two angles $\varphi_{P-1}$ and $\varphi_{P}$.

Besides, each panel is covered with an admittance $\gamma_{P}$ described by a physical model, which typically depends on a small number of parameters. From the more general study of the previous section, we realized that absorption in the surface material (through the real part of the admittance) is indeed necessary to provide the "baseline" of attenuation, but the imaginary part also plays an important part, especially if it is varying with frequency.

Following the concept of coupling dissipative and reactive impedance to broaden the frequency range of sound attenuation introduced by Namba and Fukushige [15] and further developed by Selamet et al. [16], we will consider two types of panels usually used in noise control:

- Micro-perforated resonant panel (MPP)

The surface is a sheet of metal perforated by small holes and coupled to a cavity. This type of treatment typically absorbs selected frequencies due to the resonances happening inside the cavity, but Maa [27, 28] showed that in the case of micro-perforations in the panel (less than $1 \mathrm{~mm}$ in radius), such panels can also act as absorbers on a broader frequency band.

- Absorbent layer

This type of cover is simply a layer of porous material, typically described by the Delany $\&$ Bazley model [25]. Such material provides some reasonable broadband absorption over the whole frequency range of interest. The layer version of this model has been shown to model many natural surfaces such as soils or grass relatively accurately [29, 30].

\subsection{Admittance models}

\subsubsection{Micro-perforated panel}

Such a panel is composed by sheet of a somewhat heavy material (glass or steel) of thickness $l_{0}$ and perforated by a regular grid of small holes. The hole radius is $a_{0}$ and the distance between adjacent holes is defined as $e$. However, to describe the arrangement of holes, one usually defines the porosity $s$ as the ratio of the surface covered by the holes to the total surface. Since the grid is assumed regular, this comes down to $s=\pi a_{0}^{2} / e^{2}$. This panel is mounted on top of a air cavity of depth $D$, ended with a rigid surface. This system basically behaves as a Helmholtz resonator, and therefore provides good absorption at its resonance frequencies. Also, in order to treat the MPP as locally reacting, the back cavity should be partitioned [31,32], for instance with a honeycomb structure.

Four parameters are needed to describe the acoustic behavior of this panel: $s, a_{0}, l_{0}, D$ (one could have chosen $e$ instead of $s$ ). The locally-reacting normalized impedance of the perforated panel, assuming the panel itself is rigid and one can use Sibian's model for the end correction, 
can then be written in the $\mathrm{e}^{-\mathrm{i} \omega t}$ convention as $[33,32,34]$ :

$$
\begin{aligned}
& z_{\mathrm{MPP}}(f)=-\mathrm{i} \frac{k l_{0}}{s}\left(\frac{1}{\Theta\left(x^{\prime}\right)}+\frac{16}{3 \pi} \frac{a_{0}}{l_{0}} \frac{\Psi(\xi)}{\Theta(x)}\right)+\mathrm{i} \operatorname{cotan}(k D) \\
& k=\frac{2 \pi f}{c_{0}} ; \xi=\sqrt{s} ; x=a_{0} \sqrt{\frac{2 \pi f \rho_{0}}{\mu}} ; x^{\prime}=a_{0} \sqrt{\frac{2 \pi f \rho_{0}}{\mu^{\prime}}} \\
& \mu^{\prime}=\mu\left(1+\frac{\gamma-1}{\sqrt{\operatorname{Pr}}}\right)^{2} \\
& \Theta(w)=1-\frac{2}{w \sqrt{\mathrm{i}}} \frac{J_{1}(w \sqrt{\mathrm{i}})}{J_{0}(w \sqrt{\mathrm{i}})} \\
& \Psi(\xi)=\sum_{m=0}^{8} u_{m} \xi^{m}
\end{aligned}
$$

with the coefficients $u_{m}$ given by $u_{0}=1, u_{1}=-1.4092, u_{2}=0, u_{3}=0.33818, u_{4}=0$, $u_{5}=0.06793, u_{6}=-0.02287, u_{7}=0.03015, u_{8}=-0.01641$. Physical properties of air are taken at $20^{\circ} \mathrm{C}$ : density $\rho_{0}=1.21 \mathrm{~kg} \mathrm{~m}^{-3}$, dynamic viscosity $\mu=1.8110^{-5} \mathrm{Pas}$, ratio of specific heats $\gamma=1.4$ and Prandtl number $\operatorname{Pr}=0.7$. The intermediate variables $x$ and $x^{\prime}$ are the so-called perforate constants and correspond to the ratio between the radius of the hole and either the viscous or thermal penetration depth, $\mu^{\prime}$ is an equivalent viscosity representing thermal effects. Finally, the Fok's function $[35,36] \Psi(\xi)$ is a correction to take into account interaction effects between the different holes, directly related to the porosity. The normalized admittance is then simply $\gamma_{\mathrm{MPP}}(f)=1 / z_{\mathrm{MPP}}(f)$.

\subsubsection{Rigid-backed Delany and Bazley model}

Here we consider a layer of porous material of depth $d$ ended by a rigid backing. Following Delany and Bazley [25], the normalized impedance and complex wavenumber only depend on one parameter $\sigma$ and are given by:

$$
\left\{\begin{array}{c}
\tilde{z}(f)=1+0.0511\left(\frac{\sigma}{f}\right)^{0.75}+\mathrm{i} 0.0768\left(\frac{\sigma}{f}\right)^{0.73} \\
\frac{\tilde{k}}{k}=1+0.0858\left(\frac{\sigma}{f}\right)^{0.7}+\mathrm{i} 0.175\left(\frac{\sigma}{f}\right)^{0.59}
\end{array}\right.
$$

However, because of the finite depth $d$ of the layer, and by assuming that the backing is rigid, the normalized surface impedance becomes:

$$
z_{\mathrm{DB}}(f)=\tilde{z}(f) \operatorname{coth}(-\mathrm{i} \tilde{k} d)
$$

The normalized admittance is given by $\gamma_{\mathrm{DB}}(f)=1 / z_{\mathrm{DB}}(f)$.

\subsection{Fourier series admittance coefficients and angular limit variables}

Once the panel distribution is known and the parameters for each model are determined, we end up with $N_{p}$ values of admittance depending on frequency $\gamma_{P}(f)$. To complete the description of the barrier, one still needs to specify the angular widths of each panel and therefore the $N_{p}-1$ angles $\varphi_{1}$ to $\varphi_{N_{p}-1}$ (see Fig. 9). 
Under those assumptions, the admittance distribution as a function of $\theta_{1}$ and frequency $f$ is defined as:

$$
\beta\left(\theta_{1}, f\right)=\gamma_{P}(f) \quad \text { for } \quad \theta_{1} \in\left[\varphi_{P-1}, \varphi_{P}\right] \quad P \in\left[1: N_{p}\right]
$$

Such a piecewise constant function can then be described as a Fourier series of the form given in equation (2) with the coefficients $\beta_{j}(f)$ equal to:

$$
\left\{\begin{aligned}
\beta_{0}(f) & =\frac{1}{\pi} \sum_{P=1}^{N_{p}} \gamma_{P}(f)\left(\varphi_{P}-\varphi_{P-1}\right) \\
(\forall j \geqslant 1) \quad \beta_{j}(f) & =\frac{2}{\pi j} \sum_{P=1}^{N_{p}} \gamma_{P}(f)\left[\sin \left(j \varphi_{P}\right)-\sin \left(j \varphi_{P-1}\right)\right]
\end{aligned}\right.
$$

which allows us to use the solution introduced in section 3. Of course, as before we have to cut the maximal order of the Fourier series to a finite value $N_{\max }$. We arbitrarily set it to $N_{\max }=10 N_{p}$.

\subsection{Description of the optimization problem}

Recalling Fig. 9, a finite number $N_{p}$ of panels is considered. Since we would like to use both panel types in the distribution, we will assume that the first panel extending from $\varphi_{0}=0$ to $\varphi_{1}$ (therefore further away from the source) will be a resonant panel, and that then the model type changes alternatively. For instance, if $N_{p}=4$, the panels 1 and 3 are resonant panels and the panels 2 and 4 are porous. We call a the vector of variables containing all model parameters and $\boldsymbol{\varphi}=\left(\varphi_{1}, \varphi_{2}, \varphi_{3}, \ldots\right)$ the set of angles defining the angular widths of the panels. Given $\mathbf{a}$ and $\boldsymbol{\varphi}$, there is a unique set of $N_{p}$ panel admittances $\gamma_{P}(f)$ at each frequency and therefore a unique set admittance coefficients $\beta_{j}(f)$.

Besides, in this case the frequency dependence of the admittance coefficients is intrinsically present in the admittance models, and therefore the broadband attenuation defined in section 5 can be used as the objective function.

\subsubsection{Derivatives of the objective function with respect to model parameters}

The gradient of $g$ with respect to one of the parameters used in the different admittance models is still given by equation (19) replacing $\beta_{j}$ by the corresponding coefficient.

The gradient of the attenuation $A_{q}$ with respect to the parameters a is:

$$
\frac{\partial A_{q}}{\partial \mathbf{a}}=\frac{2}{\sum_{\mathrm{i}}\left|p^{\mathrm{in}}\left(R_{\mathrm{i}}, f_{q}\right)\right|^{2}} \sum_{\mathrm{i}} \operatorname{Re}\left[p\left(R_{\mathrm{i}}, f_{q}\right)^{*}\left(\frac{\partial p_{1}^{\mathrm{sc}}\left(R_{\mathrm{i}}, f_{q}\right)}{\partial \mathbf{a}}+\frac{\partial p_{2}^{\mathrm{sc}}\left(R_{\mathrm{i}}, f_{q}\right)}{\partial \mathbf{a}}\right)\right]
$$

Furthermore, using the chain rule, one can write for $l=1,2$ :

$$
\frac{\partial p_{l}^{\mathrm{sc}}\left(R_{\mathrm{i}}, f_{q}\right)}{\partial \mathbf{a}}=\frac{\partial p_{l}^{\mathrm{sc}}\left(R_{\mathrm{i}}, f_{q}\right)}{\partial \boldsymbol{\beta}} \frac{\partial \boldsymbol{\beta}}{\partial \boldsymbol{\gamma}} \frac{\partial \boldsymbol{\gamma}\left(f_{q}\right)}{\partial \mathbf{a}}
$$

where we used the abusive notations $\partial \boldsymbol{\beta} / \partial \boldsymbol{\gamma}$ to refer to the Jacobian of the function $\boldsymbol{\gamma}(\boldsymbol{\beta})$ and $\partial \gamma\left(f_{q}\right) / \partial \mathbf{a}$ to refer to the sparse Jacobian containing all the derivatives defined in Appendix B. The gradient $\partial p_{l}^{\mathrm{sc}}\left(R_{\mathrm{i}}, f_{q}\right) / \partial \boldsymbol{\beta}$ is computed as in section 5.2. 


\subsubsection{Derivatives of the objective function with respect to angular limits}

Remains to calculate the derivative of the objective function with respect to the angle $\varphi_{P}$, $P \in\left[1: N_{p}-1\right]$. The vector $\gamma$ is independent of the angular widths, whereas the Fourier admittance coefficients $\beta$ do depend on them. Besides, since $\beta$ is a linear function of $\gamma$, one can write $\boldsymbol{\beta}=\mathbf{J} \boldsymbol{\gamma}$ where $\mathbf{J}=\partial \boldsymbol{\beta} / \partial \boldsymbol{\gamma}$ is the Jacobian introduced earlier. Finally, the derivative of the scattered field with respect to the angle $\varphi_{P}$ is given, for $l=1,2$ :

$$
\frac{\partial p_{l}^{\mathrm{sc}}\left(R_{\mathrm{i}}, f_{q}\right)}{\partial \varphi_{P}}=\frac{\partial p_{l}^{\mathrm{sc}}\left(R_{\mathrm{i}}, f_{q}\right)}{\partial \boldsymbol{\beta}} \frac{\partial \mathbf{J}}{\partial \varphi_{P}} \gamma\left(f_{q}\right)
$$

\subsubsection{Variables bounds and constraints}

To completely define the optimization problem, we still need to define the constraints the parameters and limit angles must satisfy.

First, as we mentioned in 7.2, the limit angles $\varphi_{1}$ to $\varphi_{N_{p}-1}$ of each panel are constrained to physical feasibility $\varphi_{1} \leqslant \varphi_{2} \leqslant \ldots \leqslant \varphi_{N_{p}-1}$, which can be written as a set of linear constraints. Then, admittance models parameters are constrained to be bounded by realistic lower and upper values, which depend on the type of physical parameter:

\section{- Porosity $s$}

$\overline{\text { As } s \text { goes }}$ to zero, the distance between the holes of the perforated becomes infinite, and basically the panel becomes rigid. The limit $s=0$ is therefore technically valid, but to avoid numerical problems we will set $s_{\min }=10^{-2}$. However, to ensure structural rigidity of the panel, the porosity cannot exceed a value of $s_{\max }=0.4$.

- Hole radius $a_{0}$

Again, the limit $a_{0}=0$ basically corresponds to a rigid panel as the holes shrink. Besides, when the concept of micro-perforated panel was introduced by Maa [27], it was clearly stated that to achieve a broad absorption band the radius of the holes must be less than 1 $\mathrm{mm}$. Therefore, combining this and the feasibility of the drilling process, we decided that the limit $a_{0, \min }=0.5 \mathrm{~mm}$ was reasonable. Besides, if $a_{0}$ becomes large the panel becomes transparent and the MPP globally becomes rigid because of the backing. We therefore decided to limit the value of $a_{0}$ to be less than $a_{0, \max }=5 \mathrm{~mm}$.

- Panel thickness $l_{0}$

$\overline{\text { The lower limit }}_{0}=0$ corresponds to an infinitely thin metal sheet. Again, to ensure structural integrity and a reasonable cost, we arbitrarily set the lowest value at $l_{0, \min }=2$ $\mathrm{mm}$ and $l_{0, \max }=1 \mathrm{~cm}$.

- Depth of the reverberant cavity $D$

The limit $D=0$ corresponds again to a rigid surface, and will probably be avoided by the algorithm. However, in the resonant panel model, the description of the cavity as a compliance is valid if the depth is significantly large, and therefore we chose a limit of $D_{\min }=1 \mathrm{~cm}$. Besides, if the cavity depth is large, many resonances happen in the cavity in the frequency range of interest, which might "color" the sound field in the receiver zone. Forcing a smaller value for $D$ would hence limit the number of resonances below $5000 \mathrm{~Hz}$. Empirically, we found that the value $D_{\max }=10 \mathrm{~cm}$ provides good results.

- Flow resistivity $\sigma$ This parameter is very important since the values of admittance of the porous panel strongly 
depend on it: the lower, the softer. Besides, depending on the type of materials, $\sigma$ can take a very large range of values (in $\mathrm{kPa} \mathrm{s} \mathrm{m}^{-2}$ ): from 1-10 for railway ballast, 10-30 for snow, 50-200 for outdoor ground surfaces, $800-2500$ for sandy silt, to about 30,000 for asphalt $[29,30]$. We expect the algorithm to converge to small values of the flow resistivity $\sigma$ since this would provide a better "baseline" attenuation of the barrier due to the larger absorption. However, following a sustainable development approach, we would also like to focus the search to materials that would not be environmental hazardous and that are easy to find in nature. Attenborough et al. [30] showed that many grassland-type soils can be modeled relatively accurately with a rigid-backed Delany and Bazley layer with a range of flow resistivities from $\sigma_{\min }=50 \mathrm{kPa} \mathrm{s} \mathrm{m}^{-2}$ to $\sigma_{\max }=200 \mathrm{kPas} \mathrm{m}^{-2}$. This will be the range we consider.

- Porous layer depth $d$

Also based on the results of Attenborough et al. [30], we set the extremal values of the porous layer depth to $d_{\min }=1 \mathrm{~cm}$ and $d_{\max }=10 \mathrm{~cm}$.

\subsection{Results}

The broadband attenuation defined in section 5 is minimized by changing the angles $\varphi$ and the admittance models parameters a. The optimization algorithm is again the interior-point algorithm. Furthermore, since the admittance distribution is much more constrained in this approach than in section 6, we expect efficiencies to be lower and therefore we are interested in finding a "better" optimum. We therefore used five random starting points, to keep computation time reasonable. Furthermore, the number of panels $N_{p}$ varies from 2 to 4 .

Each run of the optimization algorithm converged within a few hundreds iterations. We present the two best solutions out of the five trials we obtained for the three cases $N_{p}=2, N_{p}=3$ and $N_{p}=4$. Optimal parameters and angular widths of each panel, as well as the efficiency obtained at the end of the optimization (based on sixth-octave frequencies) and the efficiency calculated on a finer frequency scale are presented in Table 2. Obtained efficiencies for all trials are between 17.5 and $23 \mathrm{~dB}(\mathrm{~A})$, which represents an improvement compared to the rigid case of 8.5 to $14 \mathrm{~dB}(\mathrm{~A})$. Moreover, in the case $N_{p}=4$, the five trials all converged to a similar solution since obtained efficiencies are all above $22 \mathrm{~dB}(\mathrm{~A})$ and parameters values are also similar in values (the relative standard deviation of each parameter across the five trials is between 1 and $20 \%$ ).

We should also point out that the predicted insertion losses are somewhat stable, in the sense that a small variation of the angles and parameters do not induce a great decrease in the efficiency: random perturbations of $5 \%$ of all parameters in the best obtained solution induce a drop smaller than $2 \mathrm{~dB}(\mathrm{~A})$ in the efficiency.

It is interesting to notice that results are somewhat better for $N_{p}=2$ and $N_{p}=4$, which suggests that the presence of a porous absorbent panel close to the source is more effective. However, if one assumes that the semi-cylinder is covered only with a porous layer of smallest resistivity $\sigma=\sigma_{\min }$ the efficiency obtained is $15.3 \mathrm{~dB}(\mathrm{~A})$, which is lower than all the obtained solutions. Therefore, there is a definite benefit of coupling porous layers and resonant panels, which could be of the order of $8 \mathrm{~dB}(\mathrm{~A})$. Besides, substituting panels 1 and 2 by rigid panels in the best obtained solution in the case $N_{p}=4$ induce a slight change of $0.1 \mathrm{~dB}(\mathrm{~A})$ in the broadband attenuation, which suggests that the admittance on the receiver side (the side opposite to the source) does not influence the broadband insertion loss significantly.

Also, comparison of the best obtained solution with several references cases (uniformly rigid, soft or porous) shows that the improvement of the barrier covered by the four optimized panels 


\begin{tabular}{c||c||c|c|c|c|c|c}
\multicolumn{1}{c||}{ Panel } & Parameter & \multicolumn{2}{c}{$N_{p}=2$} & \multicolumn{2}{c|}{$N_{p}=3$} & \multicolumn{2}{c}{$N_{p}=4$} \\
\hline \hline \multirow{4}{*}{ \# 1 (MPP) } & Porosity $s$ & 0.27 & 0.11 & 0.15 & 0.16 & 0.37 & 0.26 \\
& Hole radius $a_{0}[\mathrm{~mm}]$ & 0.82 & 3.27 & 2.65 & 2.28 & 4.46 & 4.48 \\
& Panel thickness $l_{0}[\mathrm{~cm}]$ & 0.48 & 0.66 & 0.44 & 0.44 & 0.25 & 0.41 \\
& Cavity depth $D[\mathrm{~cm}]$ & 9.73 & 9.40 & 8.55 & 8.91 & 9.62 & 8.34 \\
& Angular width $[\times \pi \mathrm{rad}]$ & 0.62 & 0.70 & 0.34 & 0.49 & 0.14 & 0.16 \\
\hline \multirow{3}{*}{ \# 2 (porous) } & Flow resistivity $\sigma\left[\mathrm{kPa} \mathrm{s} \mathrm{m}{ }^{-2}\right]$ & 50.6 & 54.9 & 59.7 & 122.1 & 119.7 & 120.0 \\
& Layer thickness $d[\mathrm{~cm}]$ & 6.65 & 6.56 & 6.10 & 5.72 & 5.35 & 5.34 \\
& Angular width $[\times \pi \mathrm{rad}]$ & 0.38 & 0.30 & 0.07 & 0.06 & 0.09 & 0.06 \\
\hline \multirow{4}{*}{ \# 3 (MPP) } & Porosity $s$ & - & - & 0.14 & 0.17 & 0.10 & 0.12 \\
& Hole radius $a_{0}[\mathrm{~mm}]$ & - & - & 0.51 & 1.14 & 0.54 & 0.54 \\
& Panel thickness $l_{0}[\mathrm{~cm}]$ & - & - & 0.79 & 0.65 & 0.71 & 0.70 \\
& Cavity depth $D[\mathrm{~cm}]$ & - & - & 8.15 & 7.05 & 8.35 & 9.38 \\
& Angular width $[\times \pi \mathrm{rad}]$ & - & - & 0.59 & 0.45 & 0.54 & 0.54 \\
\hline \multirow{2}{*}{ \# 4 (porous) } & Flow resistivity $\sigma\left[\mathrm{kPa} \mathrm{s} \mathrm{m}{ }^{-2}\right]$ & - & - & - & - & 51.5 & 51.9 \\
& Layer thickness $d[\mathrm{~cm}]$ & - & - & - & - & 6.54 & 6.04 \\
& Angular width $[\times \pi \mathrm{rad}]$ & - & - & - & - & 0.23 & 0.23 \\
\hline \hline
\end{tabular}

Table 2: Model parameters and angular widths of each panel, as well broadband insertion losses for the two best solutions obtained by the optimization for $N_{p}=2,3,4$.

is mostly located in the mid-frequency range $300-1200 \mathrm{~Hz}$ where the source has most of its frequency content (see Fig. 10).

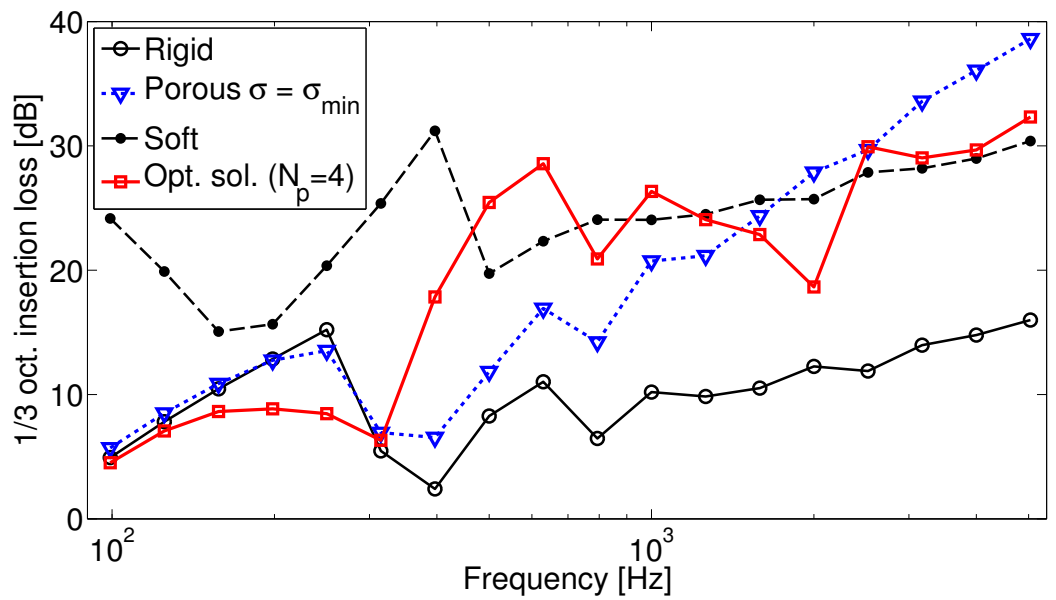

Figure 10: Third-octave insertion losses in $\mathrm{dB}$ of the best solution and comparison with reference cases: rigid $\beta=0$ (thin solid line) - uniform porous layer $\sigma=50 \mathrm{kPa} \mathrm{s} \mathrm{m}^{-2}, d=20 \mathrm{~cm}$ (dotted line) - $\operatorname{soft} \beta=\infty$ (dashed line) - optimal solution at $N_{p}=4$ (thick solid line).

Finally, one can study the correlation of the attenuation with respect to different panels ab- 
sorption coefficients. For the case of the optimal solution with $N_{p}=4$, we will focus on the two panels closest to the source - panels 3 (MPP) and 4 (porous) - since they cover most of the barrier (77\% of the surface). Plotting the absorption coefficient of those panels on top of the third-octave insertion losses (see Fig. 11), one can notice that the range of significant improvement of the attenuation $300-1200 \mathrm{~Hz}$ indeed corresponds to the first absorption band of the resonant panel. However, there does not seem to be a direct relationship between absorption and increase in attenuation, since there is a decrease in efficiency in the $315 \mathrm{~Hz}$ and $2000 \mathrm{~Hz}$ bands, both happening right before a resonance peak, which is also where the imaginary part of the MPP admittance is strongly negative (before changing sign at resonance). We therefore believe that in some circumstances, interference effects, more than energy absorption, can be responsible for the increase or decrease of the attenuation in the receiver zone, depending on the sign of the imaginary part of the resonant panel admittance. This is in agreement with the findings of section 6.

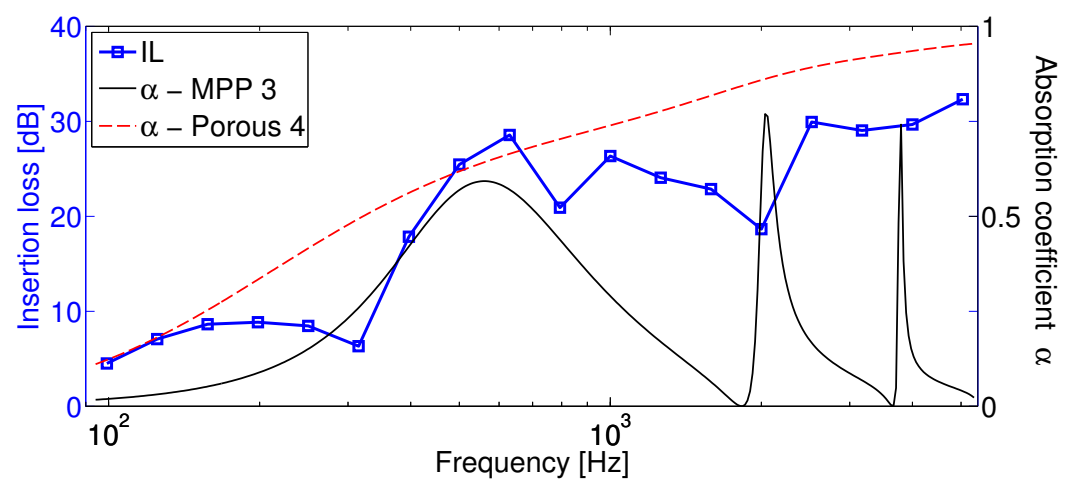

Figure 11: Comparison of the third-octave insertion losses in $\mathrm{dB}$ (thick solid line) with the absorption coefficients of the two panels close to the source MPP 3 (solid line) and Porous 4 (dotted line) at the best obtained solution with $N_{p}=4$. Parameters of the two panels are : MPP $3-s=0.123, a_{0}=0.54 \mathrm{~mm}, l_{0}=7.0 \mathrm{~mm}, D=9.4 \mathrm{~cm} ;$ Porous $4-\sigma=52$ $\mathrm{kPa} \mathrm{s} \mathrm{m}^{-2}, d=6 \mathrm{~cm}$.

\section{Conclusion}

A semi-analytical solution has been developed and validated in order to calculate the sound attenuation due to a semi-cylindrical noise barrier covered by an arbitrary distribution of admittance in the presence of a vertical baffle and a rigid ground. Such a mathematical representation is used to model the sound field close to a tramway, in presence of a semi-cylindrical low height barrier between the source and the receivers. The broadband insertion loss can be computed more efficiently than using purely numerical methods such as the BEM. The fast evaluation of the efficiency as well as its gradient with respect to the admittance distribution therefore allows a convenient optimization of the admittance distribution on the surface of the barrier.

Optimized, mathematically "ideal" distributions have been found, under the constraint that the hypothetical material creating the admittance can only absorb acoustic energy. From this theoretical study, we found that, whereas the real part of the admittance tends to be fairly constant with frequency and space in the optimal solutions, the imaginary part of the admittance undergo strong variations as a function of frequency and space, which suggests that the reactive part of 
the admittance and therefore phase-related interference effects - which are precisely very sensitive to frequency and space - can be significant to the good performance of the barrier in some circumstances. However, the absorption effect of the admittance (related to its real part) is still important to provide the baseline of the attenuation. Finally, the obtained solutions provide between 10 and $20 \mathrm{~dB}(\mathrm{~A})$ of broadband improvement over a purely rigid barrier. As for the benefit of using a non uniform admittance, we evaluated it to of the order of $8 \mathrm{~dB}(\mathrm{~A})$ of improvement.

We also applied the same scattering solution to optimize a more constrained and realistic surface admittance distribution on the semi-cylinder. We considered a barrier covered with a finite number of panels. Each of these panels is either a layer of porous material (described by the Delany and Bazley model) or a micro-perforated resonant panel. Assuming that the panels were arranged alternatively and allowing their angular width to vary, we found an improvement up to $14 \mathrm{~dB}(\mathrm{~A})$ in the optimization process compared to a purely rigid barrier. We also confirmed with this study that an important parameter is the flow resistivity of the porous layers since most optimization trials converged to small values, providing the baseline absorption that has been previously mentioned. However, we also found out that using a uniform absorbent layer with a low flow resistivity is not the optimal solution in terms of broadband efficiency. Coupling this absorbing layer with a tuned resonant panel can significantly improve the attenuation by about $8 \mathrm{~dB}(\mathrm{~A})$. Also, it seems that the attenuation is somewhat insensitive to the barrier admittance on the opposite side of the source.

Efficiencies obtained in this study are probably much higher than what would be observed in practice due to the several simplifying assumptions (rigid ground, infinite baffle, coherent line source). However, the development of the semi-analytical model that follows from those assumptions provides an efficient way to automatically choose treatment surface design parameters in order to improve the performance of the low height barrier. Actual performance of the proposed design should then be more thoroughly tested.

Furthermore, the solutions we came up with, even though showing a significant improvement in efficiency, are very unlikely to be global optima since the algorithm we used is designed to find local minima only. As shown in the theoretical study, there is potentially some more improvement to be made in the field of optimal impedance designs of noise barriers. Some other realistic designs could therefore yield even better solutions, as well as other types of surfaces which are not correctly modeled as a Delany and Bazley porous layer or a resonant micro-perforated panel. 
[1] Z. Maekawa, Noise reduction by screens, Applied Acoustics 1 (1968) 157-173.

[2] D. C. Hothersall, S. N. Chandler-Wilde, M. N. Hajmirzae, Efficiency of single noise barriers, Journal of Sound and Vibration 146 (1991) 303-322.

[3] T. Ishizuka, K. Fujiwara, Performance of noise barriers with various edge shapes and acoustical conditions, Applied Acoustics 65 (2004) 125-141.

[4] I. Ekici, H. Bougdah, A review of research on environmental noise barriers, Building Acoustics 10 (2003) $289-323$.

[5] M. Baulac, Optimisation des Protections Anti-bruit Routières de Forme Complexe (Optimization of Complex Traffic Noise Barriers), Ph.D. thesis, Université du Maine (Le Mans, France), 2006.

[6] M. Baulac, J. Defrance, Optimisation with genetic algorithm of the acoustic performance of T-shaped noise barriers with a reactive top surface, Applied Acoustics 69 (2008) 332-342.

[7] M. Möser, R. Volz, Improvement of sounds barriers using headpieces with finite acoustic impedance, Journal of the Acoustical Society of America 106 (1999) 3049-3060.

[8] T. Obuko, K. Fujiwara, Efficiency of a noise barrier on the ground with an acoustically soft cylindrical edge, Journal of Sound and Vibration 98 (1998) 771-790.

[9] T. Obuko, K. Fujiwara, Efficiency of a noise barrier with an acoustically soft cylindrical edge for practical use, Journal of the Acoustical Society of America 105 (1999) 3326-3335.

[10] D. Duhamel, Shape optimization of noise barriers using genetic algorithms, Journal of Sound and Vibration 297 (2006) 432-443.

[11] M. Baulac, J. Defrance, P. Jean, Optimization of low height noise protections in urban areas, in: Forum Acousticum 2005, Budapest, pp. 1075-1080.

[12] M. Baulac, J. Defrance, P. Jean, F. Minard, Efficiency of noise protections in urban areas: prediction and scale model measurements, Acta Acustica United with Acustica 92 (2006) 530-539.

[13] P. J. Thorsson, Optimisation of low-height noise barriers using the equivalent sources method, Acta Acustica 86 (2000) 811-820.

[14] P. J. Thorsson, Combined effects of admittance optimisation on both barrier and ground, Applied Acoustics 64 (2003) 693-711.

[15] M. Namba, K. Fukushige, Application of the equivalent source method to the acoustics of duct systems with non-uniform wall impedance, Journal of Sound and Vibration 73 (1980) 125-146.

[16] A. Selamet, I. J. Lee, N. T. Huff, Acoustic attenuation of hybrid silencers, Journal of Sound and Vibration 262 (2003) 509-527.

[17] J. T. Du, W. L. Li, Z. G. Liu, H. A. Xu, Z. L. Ji, Acoustic analysis of a rectangular cavity with general impedance boundary condition, Journal of the Acoustical Society of America 130 (2011) 807-817.

[18] M. A. Pallas, J. Lelong, R. Chatagnon, Characterization of tram noise emission and contribution of the noise sources, Applied Acoustics 72 (2011) 437-450.

[19] D. Duhamel, Efficient calculation of the three-dimensional sound pressure field around a noise barrier, Journal of Sound and Vibration 197 (1996) 547-571.

[20] D. C. Chandler-Wilde S. N., Hothersall, Efficient calculation of the green function for acoustic propagation above an impedance plane, Journal of Sound and Vibration 180 (1995) 705-724.

[21] D. A. Hutchins, H. W. Jomes, L. T. Russell, Model studies of barrier performance in the presence of ground surfaces. part ii - different shapes, Journal of the Acoustical Society of America 75 (1984).

[22] T. J. Cavicchi, W. D. J. O'Brien, Acoustic scattering of an incident cylindrical wave by an infinite circular cylinder, IEEE Transactions on Ultrasonics, Ferroelectrics and Frequency Control 35 (1988) 78-80.

[23] M. Abramovitz, I. A. Stegun, Handbook of mathematical functions, National Bureau of Standards, Applied Mathematics, 1964

[24] P. Jean, A variational approach for the study of outdoor sound propagation and application to railway noise, Journal of Sound and Vibration 212 (1998) 275-294

[25] M. E. Delany, E. N. Bazley, Acoustical properties of fibrous absorbent materials, Applied Acoustics 3 (1970) 105-116.

[26] J. Nocedal, J. Wright, S, Numerical optimization, Springer, 2006.

[27] D.-Y. Maa, Theory and design of microperforated panel sound-absorbing constructions, Scientia Sinica 18 (1975) $55-71$

[28] D.-Y. Maa, Potential of microperforated panel absorber, Journal of the Acoustical Society of America 104 (1998) 2861-2866.

[29] K. Attenborough, K. M. Li, K. Horoshenkov, Predicting outdoor sound, Taylor and Francis, 2007.

[30] K. Attenborough, I. Bashir, S. Taherzadeh, Outdoor ground impedance models, Journal of the Acoustical Society of America 129 (2011) 2806-2819.

[31] M. Q. Wu, Micro-perforated panels for duct silencing, Noise Control Engineering Journal 45 (1997) 69-77.

[32] F. Asdrubali, G. Pispola, Properties of transparent sound-absorbing panels for use in noise barriers, Journal of the Acoustical Society of America 121 (2007) 214-221. 
[33] T. H. Melling, The acoustic impedance of perforates at medium and high sound pressure levels, Journal of Sound and Vibration 29 (1975) 1-65.

[34] A. F. Sobolev, A semi-empirical theory of a one-layer cellular sound-absorbing lining with a perforated face panel, Akusticheskii Zhurnal (Soviet Physics-Acoustics Journal) 53 (2007) 762-771.

[35] V. A. Fok, Teoreticheskoe issledovanie provodimosti kruglogo otverstiya v peregorodke, postavlennoi poperek truby (theoretical study of the conductance of a circular hole in a partition across a tube), Doklady Akademii Nauk SSSR (Soviet Physics Doklady) 31 (1941) 875-882.

[36] S. N. Rzhevkin, A course of lectures on the theory of sound, Pergamon Press, London, 1963. 
Appendix A. Derivation of the system of equations satisfied by the scattered field coefficients

Appendix A.1. Boundary condition on the true cylinder

Determination of the matrix equation which determines the scattered fields coefficients $\alpha_{n}^{1}$ and $\alpha_{n}^{2}$ comes from the application of the two boundary conditions (1) and (3). Let us first detail the condition on the true cylinder $r_{1}=a$ :

$$
\begin{aligned}
& \underbrace{\frac{1}{k} \frac{\partial p_{1}^{\mathrm{sc}}}{\partial r_{1}}\left(a, \theta_{1}\right)+\mathrm{i} \beta\left(\theta_{1}\right) p_{1}^{\mathrm{sc}}\left(a, \theta_{1}\right)}_{=\mathcal{A}_{1}}+\underbrace{\frac{1}{k} \frac{\partial p_{2}^{\mathrm{sc}}}{\partial r_{1}}\left(a, \theta_{1}\right)+\mathrm{i} \beta\left(\theta_{1}\right) p_{2}^{\mathrm{sc}}\left(a, \theta_{1}\right)}_{=\mathcal{B}_{1}} \\
= & \underbrace{-\frac{1}{k} \frac{\partial p^{\mathrm{in}}}{\partial r_{1}}\left(a, \theta_{1}\right)-\mathrm{i} \beta\left(\theta_{1}\right) p^{\mathrm{in}}\left(a, \theta_{1}\right)}_{=C_{1}}
\end{aligned}
$$

where both sides of the boundary condition have been divided by $k$ for convenience. $\mathcal{A}_{1}$ corresponds to the influence of $p_{1}^{\mathrm{sc}}$ on the true cylinder, $\mathcal{B}_{1}$ corresponds to the influence of $p_{2}^{\mathrm{sc}}$ on the true cylinder (coupling term) and $C_{1}$ corresponds to the forcing due to the incident field (right hand side). Each of these terms can be expanded as a Fourier series in $\theta_{1}$ parametrized by $p$ and identification of those coefficients yields each line of the system (11). Because in the end we want the different expressions to be expressed as Fourier series in $\theta_{1}$ parametrized by the index $p$, whenever a sum can be directly identified as such a series, the index will be changed into $p$.

Appendix A.1.1. Right hand side: term $C_{1}$

Substitutions of the expressions of $p^{\text {in }}$ given in equation (7) and $\beta$ given in equation (2) yield:

$$
\begin{aligned}
C_{1}=- & \frac{1}{k} \frac{\partial p^{\mathrm{in}}}{\partial r_{1}}\left(a, \theta_{1}\right)-\mathrm{i} \beta\left(\theta_{1}\right) p^{\mathrm{in}}\left(a, \theta_{1}\right) \\
=- & \frac{\mathrm{i}}{2} J_{0}^{\prime}(k a) H_{0}^{(1)}(k d)-\mathrm{i} \sum_{m=1}^{\infty} J_{m}^{\prime}(k a) H_{m}^{(1)}(k d) \cos \left(m \theta_{s}\right) \cos \left(m \theta_{1}\right) \\
& +\frac{1}{2} J_{0}(k a) H_{0}^{(1)}(k d) \sum_{j=0}^{\infty} \beta_{j} \cos \left(j \theta_{1}\right) \\
& +\sum_{m=1}^{\infty} \sum_{j=0}^{\infty} J_{m}(k a) H_{m}^{(1)}(k d) \beta_{j} \cos \left(m \theta_{s}\right) \cos \left(m \theta_{1}\right) \cos \left(j \theta_{1}\right) \\
=- & \frac{\mathrm{i}}{2} J_{0}^{\prime}(k a) H_{0}^{(1)}(k d)-\mathrm{i} \sum_{p=1}^{\infty} J_{p}^{\prime}(k a) H_{p}^{(1)}(k d) \cos \left(p \theta_{s}\right) \cos \left(p \theta_{1}\right) \\
& +\frac{1}{2} \sum_{p=0}^{\infty} J_{0}(k a) H_{0}^{(1)}(k d) \beta_{p} \cos \left(p \theta_{1}\right)+\frac{A}{2}+\frac{B}{2}
\end{aligned}
$$


where the formula $\cos \left(m \theta_{1}\right) \cos \left(j \theta_{1}\right)=\left(\cos \left[(m+j) \theta_{1}\right]+\cos \left[(m-j) \theta_{1}\right]\right) / 2$ has been used so that $A$ and $B$ are:

$$
\begin{aligned}
& A=\sum_{m=1}^{\infty} \sum_{j=0}^{\infty} J_{m}(k a) H_{m}^{(1)}(k d) \cos \left(m \theta_{s}\right) \beta_{j} \cos \left[(m+j) \theta_{1}\right] \\
& B=\sum_{m=1}^{\infty} \sum_{j=0}^{\infty} J_{m}(k a) H_{m}^{(1)}(k d) \cos \left(m \theta_{s}\right) \beta_{j} \cos \left[(m-j) \theta_{1}\right]
\end{aligned}
$$

Since our goal is to identify the Fourier series coefficient between the two sides of equation (A.1), we need to change the variables $(m, n)$ in the two double sums to isolate either $p=m+j$ or $p=m-j$. Considering $A$, this yields:

$$
\begin{aligned}
A & =\sum_{p=1}^{\infty} \cos \left(p \theta_{1}\right) \sum_{m+j=p} J_{m}(k a) H_{m}^{(1)}(k d) \cos \left(m \theta_{s}\right) \beta_{j} \\
& =\sum_{p=1}^{\infty} \cos \left(p \theta_{1}\right) \sum_{j=0}^{p-1} J_{p-j}(k a) H_{p-j}^{(1)}(k d) \cos \left[(p-j) \theta_{s}\right] \beta_{j} \\
& =\sum_{p=1}^{\infty} \cos \left(p \theta_{1}\right) \sum_{j=0}^{p-1} J_{-p+j}(k a) H_{-p+j}^{(1)}(k d) \cos \left[(-p+j) \theta_{s}\right] \beta_{j}
\end{aligned}
$$

where we used the property $W_{-r}=(-1)^{r} W_{r}$ for any integer order $r$ Bessel functions and the even symmetry of cos.

The parametrization in $B$ is more complicated since $p$ goes from $-\infty$ to $+\infty$. Breaking up $B$ in three terms $p=0, p>0$ and $p<0$, one can write:

$$
\begin{aligned}
& B= \sum_{p=-\infty}^{\infty} \cos \left(p \theta_{1}\right) \sum_{m-j=p} J_{m}(k a) H_{m}^{(1)}(k d) \cos \left(m \theta_{s}\right) \beta_{j} \\
&=\sum_{j=1}^{\infty} J_{j}(k a) H_{j}^{(1)}(k d) \cos \left(j \theta_{s}\right) \beta_{j} \\
& \quad+\sum_{p=-\infty}^{-1} \cos \left(p \theta_{1}\right) \sum_{j=-p+1}^{\infty} J_{p+j}(k a) H_{p+j}^{(1)}(k d) \cos \left[(p+j) \theta_{s}\right] \beta_{n} \\
& \quad+\sum_{p=1}^{\infty} \cos \left(p \theta_{1}\right) \sum_{j=0}^{\infty} J_{p+j}(k a) H_{p+j}^{(1)}(k d) \cos \left[(p+j) \theta_{s}\right] \beta_{j} \\
&=\sum_{j=1}^{\infty} J_{j}(k a) H_{j}^{(1)}(k d) \cos \left(j \theta_{s}\right) \beta_{j} \\
& \quad+\sum_{p=1}^{\infty} \cos \left(p \theta_{1}\right) \sum_{j=p+1}^{\infty} J_{-p+j}(k a) H_{-p+j}^{(1)}(k d) \cos \left[(-p+j) \theta_{s}\right] \beta_{j} \\
& \quad+\sum_{p=1}^{\infty} \cos \left(p \theta_{1}\right) \sum_{j=0}^{\infty} J_{p+j}(k a) H_{p+j}^{(1)}(k d) \cos \left[(p+j) \theta_{s}\right] \beta_{j}
\end{aligned}
$$


where we rewrote the second sum changing $p$ into $-p$ and using the even symmetry of cos so that only positive values of the index $p$ are used.

Recalling equation (A.2) and noticing that we can combine $A$, the second sum of $B$ and the term $\sum_{j=0}^{\infty} J_{0}(k a) H_{0}^{(1)}(k d) \beta_{j} \cos \left(j \theta_{1}\right)$ into one sum, we end up with:

$$
\begin{aligned}
& C_{1}=- \frac{\mathrm{i}}{2} J_{0}^{\prime}(k a) H_{0}^{(1)}(k d)+\frac{1}{2} \sum_{j=1}^{\infty} J_{j}(k a) H_{j}^{(1)}(k d) \cos \left(j \theta_{s}\right) \beta_{j} \\
&-\mathrm{i} \sum_{p=1}^{\infty} J_{p}^{\prime}(k a) H_{p}^{(1)}(k d) \cos \left(p \theta_{s}\right) \cos \left(p \theta_{1}\right) \\
&+\frac{1}{2} \sum_{p=1}^{\infty} \cos \left(p \theta_{1}\right) \sum_{j=0}^{\infty} J_{p+j}(k a) H_{p+j}^{(1)}(k d) \cos \left[(p+j) \theta_{s}\right] \beta_{j} \\
&+\frac{1}{2} \sum_{p=1}^{\infty} \cos \left(p \theta_{1}\right) \sum_{j=0}^{\infty} J_{-p+j}(k a) H_{-p+j}^{(1)}(k d) \cos \left[(-p+j) \theta_{s}\right] \beta_{j} \\
&=\sum_{p=0}^{\infty} e_{p}^{1} \cos \left(p \theta_{1}\right)
\end{aligned}
$$

where the coefficients $e_{p}^{1}$ are defined as in equation (12).

Appendix A.1.2. Coupling of $p_{1}^{s c}$ with the true cylinder: term $\mathcal{A}_{1}$

Substitution of $p_{1}^{\mathrm{sc}}$ and $\beta$ by their series expansions given in equations (6) and (2) yields:

$$
\begin{aligned}
\mathcal{A}_{1}= & \frac{1}{k} \frac{\partial p_{1}^{\mathrm{sc}}}{\partial r_{1}}\left(a, \theta_{1}\right)+\mathrm{i} \beta\left(\theta_{1}\right) p_{1}^{\mathrm{sc}}\left(a, \theta_{1}\right) \\
= & \frac{\alpha_{0}^{1}}{2}\left(\frac{H_{0}^{(1)^{\prime}}(k a)}{H_{0}^{(1)}(k a)}+\mathrm{i} \beta_{0}\right)+\sum_{n=1}^{\infty} \alpha_{n}^{1} \frac{H_{n}^{(1)^{\prime}}(k a)}{H_{n}^{(1)}(k a)} \cos \left(n \theta_{1}\right)+\frac{\mathrm{i}}{2} \sum_{j=1}^{\infty} \alpha_{0}^{1} \beta_{j} \cos \left(j \theta_{1}\right) \\
& +\mathrm{i} \sum_{j=0}^{\infty} \sum_{n=1}^{\infty} \alpha_{n}^{1} \beta_{j} \cos \left(j \theta_{1}\right) \cos \left(n \theta_{1}\right) \\
= & \frac{\alpha_{0}^{1}}{2}\left(\frac{H_{0}^{(1)^{\prime}}(k a)}{H_{0}^{(1)}(k a)}+\mathrm{i} \beta_{0}\right)+\sum_{p=1}^{\infty} \alpha_{p}^{1} \frac{H_{p}^{(1)^{\prime}}(k a)}{H_{p}^{(1)}(k a)} \cos \left(p \theta_{1}\right)+\frac{\mathrm{i}}{2} \sum_{p=1}^{\infty} \alpha_{0}^{1} \beta_{p} \cos \left(p \theta_{1}\right)+\frac{\mathrm{i}}{2} C
\end{aligned}
$$

with $C$ defined by:

$$
C=\sum_{j=0}^{\infty} \sum_{n=1}^{\infty} \alpha_{n}^{1} \beta_{j} \cos \left[(n+j) \theta_{1}\right]+\sum_{j=0}^{\infty} \sum_{n=1}^{\infty} \alpha_{n}^{1} \beta_{j} \cos \left[(n-j) \theta_{1}\right]
$$


We perform the same change of variables in the double sum, except that for each value of $p$, we will sum on $n$ in order to have a simple dependence on the unknown coefficients $\alpha_{n}^{1}$. This yields:

$$
\begin{aligned}
& C=\sum_{p=1}^{\infty} \cos \left(p \theta_{1}\right) \sum_{n=1}^{p} \alpha_{n}^{1} \beta_{p-n}+\sum_{n=1}^{\infty} \alpha_{n}^{1} \beta_{n}+\sum_{p=1}^{\infty} \cos \left(p \theta_{1}\right) \sum_{n=p}^{\infty} \alpha_{n}^{1} \beta_{n-p} \\
&+ \underbrace{\sum_{p=0}^{-1} \cos \left(p \theta_{1}\right) \sum_{n=1}^{\infty} \alpha_{n}^{1} \beta_{n-p}}_{p=-\infty} \\
&=\sum_{p=1}^{\infty} \cos \left(p \theta_{1}\right) \sum_{n=1}^{\infty} \alpha_{n}^{1} \beta_{n+p}
\end{aligned}
$$

where again we changed $p$ into $-p$ to avoid negative values of the Fourier series index $p$. Substitution into equation (A.3) and regrouping terms yields:

$$
\begin{aligned}
\mathcal{A}_{1}= & \frac{H_{0}^{(1)^{\prime}}(k a)}{2 H_{0}^{(1)}(k a)} \alpha_{0}^{1}+\frac{\mathrm{i}}{2} \sum_{n=0}^{\infty} \alpha_{n}^{1} \beta_{n}+\sum_{p=1}^{\infty} \cos \left(p \theta_{1}\right)\left[\frac{\mathrm{i}}{2} \sum_{n=0}^{\infty} \beta_{n+p} \alpha_{n}^{1}+\left(\frac{H_{p}^{(1)^{\prime}}(k a)}{H_{p}^{(1)}(k a)}+\mathrm{i} \beta_{0}\right) \alpha_{p}^{1}\right. \\
& \left.\quad+\frac{\mathrm{i}}{2} \sum_{n=1}^{p-1} \beta_{p-n} \alpha_{n}^{1}+\frac{\mathrm{i}}{2} \sum_{n=p+1}^{\infty} \beta_{n-p} \alpha_{n}^{1}\right] \\
= & \sum_{p=0}^{\infty} \cos \left(p \theta_{1}\right) \sum_{n=0}^{\infty} \mathbf{M}_{p n}^{11} \alpha_{n}^{1}
\end{aligned}
$$

with the coefficients $\mathbf{M}_{p n}^{11}$ defined in equation (13).

Appendix A.1.3. Coupling of $p_{2}^{s c}$ with the true cylinder: term $\mathcal{B}_{1}$

Recalling equation (8) and inverting the two summations, one can write $p_{2}^{\mathrm{sc}}\left(r_{1}, \theta_{1}\right)=\sum_{m=0}^{\infty} D_{m} \cos \left(m \theta_{1}\right)$ with, for $m>0$ :

$$
\left\{\begin{array}{l}
D_{0}=\sum_{n=0}^{\infty} \frac{\alpha_{n}^{2}}{\epsilon_{n} H_{n}^{(1)}(k a)} H_{n}^{(1)}(k b) J_{0}(k a) \\
D_{m}=\sum_{n=0}^{\infty} \frac{\alpha_{n}^{2}}{\epsilon_{n} H_{n}^{(1)}(k a)}(-1)^{m}\left(H_{m+n}^{(1)}(k b)+(-1)^{n} H_{m-n}^{(1)}(k b)\right) J_{m}(k a)
\end{array}\right.
$$

Using a similar change of variables, the cross-coupling term in the boundary condition $\mathcal{B}_{1}$ becomes:

$$
\begin{aligned}
\mathcal{B}_{1} & =\sum_{m=0}^{\infty} D_{m}^{\prime} \cos \left(m \theta_{1}\right)+\mathrm{i} \sum_{m=0}^{\infty} \sum_{j=0}^{\infty} D_{m} \beta_{j} \cos \left(m \theta_{1}\right) \cos \left(j \theta_{1}\right) \\
& =\sum_{p=0}^{\infty} D_{p}^{\prime} \cos \left(p \theta_{1}\right)+\frac{\mathrm{i}}{2} \sum_{m=0}^{\infty} \sum_{j=0}^{\infty} D_{m} \beta_{j} \cos \left[(j+m) \theta_{1}\right]+\frac{\mathrm{i}}{2} \sum_{m=0}^{\infty} \sum_{j=0}^{\infty} D_{m} \beta_{j} \cos \left[(j-m) \theta_{1}\right] \\
& =\sum_{p=0}^{\infty} D_{p}^{\prime} \cos \left(p \theta_{1}\right)+\frac{\mathrm{i}}{2}\left[\sum_{p=0}^{\infty} \cos \left(p \theta_{1}\right) \sum_{m+j=p} D_{m} \beta_{j}+\sum_{p=-\infty}^{\infty} \cos \left(p \theta_{1}\right) \sum_{j-m=p} D_{m} \beta_{j}\right]
\end{aligned}
$$




$$
\begin{aligned}
& =\sum_{p=0}^{\infty} D_{p}^{\prime} \cos \left(p \theta_{1}\right)+\frac{\mathrm{i}}{2}\left[\sum_{p=0}^{\infty} \cos \left(p \theta_{1}\right) \sum_{m=0}^{p} D_{m} \beta_{p-m}+\sum_{p=0}^{\infty} \cos \left(p \theta_{1}\right) \sum_{m=0}^{\infty} D_{m} \beta_{p+m}\right] \\
& +\frac{\mathrm{i}}{2} \underbrace{\sum_{p=-1}^{-1} \cos \left(p \theta_{1}\right) \sum_{m=-p}^{\infty} D_{m} \beta_{p+m}}_{p=-\infty} \\
& \quad=\sum_{p=1}^{\infty} \cos \left(p \theta_{1}\right) \sum_{m=p}^{\infty} D_{m} \beta_{-p+m} \\
& =D_{0}^{\prime}+\mathrm{i} \beta_{0} D_{0}+\frac{\mathrm{i}}{2} \sum_{m=1}^{\infty} \beta_{m} D_{m} \\
& +\sum_{p=1}^{\infty} \cos \left(p \theta_{1}\right)\left[D_{p}^{\prime}+\frac{\mathrm{i}}{2} \sum_{m=0}^{p-1} D_{m}\left(\beta_{p-m}+\beta_{p+m}\right)+\frac{\mathrm{i}}{2} D_{p}\left(2 \beta_{0}+\beta_{2 p}\right)\right. \\
& \left.+\frac{\mathrm{i}}{2} \sum_{m=p+1}^{\infty} D_{m}\left(\beta_{m-p}+\beta_{p+m}\right)\right]
\end{aligned}
$$

with the coefficients $D_{m}^{\prime}$ defined similarly than $D_{m}$ replacing the term $J_{m}(k a)$ by $J_{m}^{\prime}(k a)$ and again changing $p$ into $-p$ in the last sum. Again, the set of equations satisfied by the coefficients $\alpha^{2}$ comes from identification of the Fourier coefficients. For $p=0$, substituting the expression for $D_{m}$ given in equation (A.4) yields:

$$
\begin{aligned}
& D_{0}^{\prime}+\mathrm{i} \beta_{0} D_{0}+\frac{\mathrm{i}}{2} \sum_{m=1}^{\infty} \beta_{m} D_{m} \\
= & \sum_{n=0}^{\infty} \frac{\alpha_{n}^{2}}{\epsilon_{n} H_{n}^{(1)}(k a)}\left[H_{n}^{(1)}(k b)\left(J_{0}^{\prime}(k a)+\mathrm{i} \beta_{0} J_{0}(k a)\right)\right. \\
& \left.\quad+\frac{\mathrm{i}}{2} \sum_{m=1}^{\infty}(-1)^{m}\left(H_{m+n}^{(1)}(k b)+(-1)^{n} H_{m-n}^{(1)}(k b)\right) J_{m}(k a) \beta_{m}\right] \\
= & \sum_{n=0}^{\infty} \mathbf{M}_{0 n}^{12} \alpha_{n}^{2}
\end{aligned}
$$

with $\mathbf{M}_{0 n}^{12}$ defined as in equation (14).

Identification of the Fourier coefficient of order $p>0$ yields:

$$
\begin{aligned}
& D_{p}^{\prime}+\frac{\mathrm{i}}{2} \sum_{m=0}^{p-1} D_{m}\left(\beta_{p-m}+\beta_{p+m}\right)+\frac{\mathrm{i}}{2} D_{p}\left(2 \beta_{0}+\beta_{2 p}\right)+\frac{\mathrm{i}}{2} \sum_{m=p+1}^{\infty} D_{m}\left(\beta_{m-p}+\beta_{p+m}\right) \\
& =\sum_{n=0}^{\infty} \frac{\alpha_{n}^{2}}{\epsilon_{n} H_{n}^{(1)}(k a)}\left[(-1)^{p}\left(J_{p}^{\prime}(k a)+\mathrm{i} \beta_{0} J_{p}(k a)+\frac{\mathrm{i}}{2} \beta_{2 p} J_{p}(k a)\right)\left(H_{n+p}^{(1)}(k b)+(-1)^{n} H_{p-n}^{(1)}(k b)\right)\right. \\
& \quad+\mathrm{i} \beta_{p} H_{n}^{(1)}(k b) J_{0}(k a) \\
& \quad+\frac{\mathrm{i}}{2} \sum_{m=1}^{p-1}\left(\beta_{p-m}+\beta_{p+m}\right)(-1)^{m} J_{m}(k a)\left(H_{n+m}^{(1)}(k b)+(-1)^{n} H_{m-n}^{(1)}(k b)\right)
\end{aligned}
$$




$$
\begin{aligned}
& \left.+\frac{\mathrm{i}}{2} \sum_{m=p+1}^{\infty}\left(\beta_{m-p}+\beta_{p+m}\right)(-1)^{m} J_{m}(k a)\left(H_{n+m}^{(1)}(k b)+(-1)^{n} H_{m-n}^{(1)}(k b)\right)\right] \\
= & \sum_{n=0}^{\infty} \mathbf{M}_{p n}^{12} \alpha_{n}^{2}
\end{aligned}
$$

with $\mathbf{M}_{p n}^{12}$ defined as in equation (14).

Appendix A.1.4. First line of the system of equations

Putting everything together, the boundary condition on the true cylinder (A.1) becomes:

$$
\sum_{p=0}^{\infty} \cos \left(p \theta_{1}\right)\left[\sum_{n=0}^{\infty} \mathbf{M}_{p n}^{11} \alpha_{n}^{1}+\sum_{n=0}^{\infty} \mathbf{M}_{p n}^{12} \alpha_{n}^{2}\right]=\sum_{p=0}^{\infty} e_{p}^{1} \cos \left(p \theta_{1}\right)
$$

By identification of each coefficient in the Fourier series in $\theta_{1}$ on both sides, we end with the set of equations:

$$
(\forall p \in \mathbb{N}) \quad \sum_{m=0}^{\infty} \mathbf{M}_{p n}^{11} \alpha_{n}^{1}+\sum_{n=0}^{\infty} \mathbf{M}_{p n}^{12} \alpha_{n}^{2}=e_{p}^{1} \quad \Leftrightarrow \quad \mathbf{M}^{11} \boldsymbol{\alpha}^{1}+\mathbf{M}^{12} \boldsymbol{\alpha}^{2}=\mathbf{e}^{1}
$$

which is the first line of the system (11).

Appendix A.2. Boundary condition on the image cylinder

Similarly, application of the boundary condition (3) on the image cylinder yields:

$$
\begin{aligned}
\underbrace{\frac{1}{k} \frac{\partial p_{1}^{\mathrm{sc}}}{\partial r_{2}}\left(a, \theta_{2}\right)+\mathrm{i} \tilde{\beta}\left(\theta_{2}\right) p_{1}^{\mathrm{sc}}\left(a, \theta_{2}\right)}_{=\mathcal{B}_{2}}+\underbrace{\frac{1}{k} \frac{\partial p_{2}^{\mathrm{sc}}}{\partial r_{2}}\left(a, \theta_{2}\right)+\mathrm{i} \tilde{\beta}\left(\theta_{2}\right) p_{2}^{\mathrm{sc}}\left(a, \theta_{2}\right)}_{=\mathcal{H}_{2}} \\
=\underbrace{-\frac{1}{k} \frac{\partial p^{\mathrm{in}} \frac{\mathrm{i}_{2}}{\partial r_{2}}\left(a, \theta_{2}\right)-\mathrm{i} \beta\left(\theta_{2}\right) p^{\mathrm{in}}\left(a, \theta_{2}\right)}{}}_{=C_{2}}
\end{aligned}
$$

Very similar calculations can be performed to expand each of those terms as a Fourier series in $\theta_{2}$, which will yield a similar set of equations. However, because of the slight differences in the series expansions of all fields $p^{\text {in }}, p_{1}^{\text {sc }}$ and $p_{2}^{\text {sc }}$, we expect the values of $\mathbf{e}^{2}, \mathbf{M}^{21}$ and $\mathbf{M}^{22}$ to be related to, but different than $\mathbf{e}^{1}, \mathbf{M}^{11}$ and $\mathbf{M}^{12}$.

\section{Appendix A.2.1. Right hand side: term $C_{2}$}

Comparing equations (7) and (9), one can notice that the only difference, when evaluated on the corresponding cylinders $r_{1}=a$ or $r_{2}=a$, is the source angle $\theta_{s}$ changed in $\pi-\theta_{s}$. Besides, on the image cylinder the admittance coefficients are simply multiplied by $(-1)^{j}$. Therefore, making those two substitutions in the definition of $e_{p}^{1}$, and using the fact that for any integer $r$ and real $w$ we have $\cos [r(\pi-w)]=(-1)^{r} \cos (r w)$, one has for $p=0$ :

$$
\begin{aligned}
e_{0}^{2} & =-\frac{\mathrm{i}}{2} J_{0}^{\prime}(k a) H_{0}^{(1)}(k d)+\frac{1}{2} \sum_{j=0}^{\infty} J_{j}(k a) H_{j}^{(1)}(k d) \cos \left[j\left(\pi-\theta_{s}\right)\right] \tilde{\beta}_{j} \\
& =-\frac{\mathrm{i}}{2} J_{0}^{\prime}(k a) H_{0}^{(1)}(k d)+\frac{1}{2} \sum_{j=0}^{\infty} J_{j}(k a) H_{j}^{(1)}(k d)(-1)^{j} \cos \left(j \theta_{s}\right)(-1)^{j} \beta_{j}=e_{0}^{1}
\end{aligned}
$$


and for $p>0$ :

$$
\begin{aligned}
e_{p}^{2}=-\mathrm{i} & J_{p}^{\prime}(k a) H_{p}^{(1)}(k d) \cos \left[p\left(\pi-\theta_{s}\right)\right] \\
& +\frac{1}{2} \sum_{j=0}^{\infty} \tilde{\beta}_{j} J_{j-p}(k a) H_{j-p}^{(1)}(k d) \cos \left[(j-p)\left(\pi-\theta_{s}\right)\right] \\
& +\frac{1}{2} \sum_{j=0}^{\infty} \tilde{\beta}_{j} J_{j+p}(k a) H_{j+p}^{(1)}(k d) \cos \left[(j+p)\left(\pi-\theta_{s}\right)\right] \\
=-\mathrm{i} & J_{p}^{\prime}(k a) H_{p}^{(1)}(k d)(-1)^{p} \cos \left(p \theta_{s}\right) \\
& +\frac{1}{2} \sum_{j=0}^{\infty}(-1)^{j} \beta_{j} J_{j-p}(k a) H_{j-p}^{(1)}(k d)(-1)^{j-p} \cos \left[(j-p) \theta_{s}\right] \\
& +\frac{1}{2} \sum_{j=0}^{\infty}(-1)^{j} \beta_{j} J_{j+p}(k a) H_{j+p}^{(1)}(k d)(-1)^{j+p} \cos \left[(j+p) \theta_{s}\right] \\
=(-1)^{p} & e_{p}^{1}
\end{aligned}
$$

Appendix A.2.2. Coupling of $p_{2}^{s c}$ with the image cylinder: term $\mathcal{A}_{2}$

The only difference between $\mathcal{A}_{2}$ and $\mathcal{A}_{1}$ comes from the symmetrical admittance distribution $\tilde{\beta}$. Therefore, one can directly write:

$$
\begin{aligned}
\mathcal{A}_{2}= & \frac{H_{0}^{(1)^{\prime}}(k a)}{2 H_{0}^{(1)}(k a)} \alpha_{0}^{1}+\frac{\mathrm{i}}{2} \sum_{n=0}^{\infty} \alpha_{n}^{1}(-1)^{n} \beta_{n}+\sum_{p=1}^{\infty} \cos \left(p \theta_{1}\right)\left[\frac{\mathrm{i}}{2} \sum_{n=0}^{\infty}(-1)^{n+p} \beta_{n+p} \alpha_{n}^{1}\right. \\
& \left.\quad+\left(\frac{H_{p}^{(1)^{\prime}}(k a)}{H_{p}^{(1)}(k a)}+\mathrm{i} \beta_{0}\right) \alpha_{p}^{1}+\frac{\mathrm{i}}{2} \sum_{n=1}^{p-1}(-1)^{p-n} \beta_{p-n} \alpha_{n}^{1}+\frac{\mathrm{i}}{2} \sum_{n=p+1}^{\infty}(-1)^{n-p} \beta_{n-p} \alpha_{n}^{1}\right] \\
= & \sum_{p=0}^{\infty} \cos \left(p \theta_{2}\right) \sum_{n=0}^{\infty} \mathbf{M}_{p n}^{22} \alpha_{n}^{2}
\end{aligned}
$$

Appendix A.2.3. Coupling of $p_{1}^{s c}$ with the image cylinder: term $\mathcal{B}_{2}$

Recalling equation (10) and following the same approach than in section Appendix A.1, one can write $p_{1}^{\mathrm{sc}}$ evaluated at $r_{2}=a$ as $p_{1}^{\mathrm{sc}}\left(a, \theta_{2}\right)=\sum_{m=0}^{\infty} E_{m} \cos \left(m \theta_{2}\right)$ with (for $\left.m>0\right)$ :

$$
\left\{\begin{array}{l}
E_{0}=\sum_{n=0}^{\infty} \frac{\alpha_{n}^{1}}{\epsilon_{n} H_{n}^{(1)}(k a)}(-1)^{n} H_{n}^{(1)}(k b) J_{0}(k a) \\
E_{m}=\sum_{n=0}^{\infty} \frac{\alpha_{n}^{1}}{\epsilon_{n} H_{n}^{(1)}(k a)}\left((-1)^{n} H_{m+n}^{(1)}(k b)+H_{m-n}^{(1)}(k b)\right) J_{m}(k a)
\end{array}\right.
$$


Using the same approach, the cross-coupling term in the boundary condition becomes:

$$
\begin{aligned}
\mathcal{B}_{2}= & E_{0}^{\prime}+\mathrm{i} \beta_{0} E_{0}+\frac{\mathrm{i}}{2} \sum_{m=1}^{\infty}(-1)^{m} \beta_{m} E_{m} \\
& +\sum_{p=1}^{\infty} \cos \left(p \theta_{1}\right)\left[E_{p}^{\prime}+\frac{\mathrm{i}}{2} \sum_{m=0}^{p-1} E_{m}\left((-1)^{p-m} \beta_{p-m}+(-1)^{p+m} \beta_{p+m}\right)+\frac{\mathrm{i}}{2} E_{p}\left(2 \beta_{0}+\beta_{2 p}\right)\right. \\
& \left.+\frac{\mathrm{i}}{2} \sum_{m=p+1}^{\infty} E_{m}\left((-1)^{m-p} \beta_{m-p}+(-1)^{p+m} \beta_{p+m}\right)\right]
\end{aligned}
$$

with the coefficients $E_{m}^{\prime}$ defined in a similar fashion than in Appendix A.1. The term $p=0$ is:

$$
\begin{aligned}
& E_{0}^{\prime}+\mathrm{i} \beta_{0} E_{0}+\frac{\mathrm{i}}{2} \sum_{m=1}^{\infty}(-1)^{m} \beta_{m} E_{m} \\
& =\sum_{n=0}^{\infty} \frac{\alpha_{n}^{1}}{\epsilon_{n} H_{n}^{(1)}(k a)}\left[(-1)^{n} H_{n}^{(1)}(k b)\left(J_{0}^{\prime}(k a)+\mathrm{i} \beta_{0} J_{0}(k a)\right)+\right. \\
& \left.+\frac{\mathrm{i}}{2} \sum_{m=1}^{\infty}\left((-1)^{n} H_{m+n}^{(1)}(k b)+H_{m-n}^{(1)}(k b)\right) J_{m}(k a)(-1)^{m} \beta_{m}\right] \\
& =\sum_{n=0}^{\infty}(-1)^{n} \frac{\alpha_{n}^{1}}{\epsilon_{n} H_{n}^{(1)}(k a)}\left[H_{n}^{(1)}(k b)\left(J_{0}^{\prime}(k a)+\mathrm{i} \beta_{0} J_{0}(k a)\right)\right. \\
& \left.+\frac{\mathrm{i}}{2} \sum_{m=1}^{\infty}(-1)^{m}\left(H_{m+n}^{(1)}(k b)+(-1)^{n} H_{m-n}^{(1)}(k b)\right) J_{m}(k a) \beta_{m}\right] \\
& =\sum_{n=0}^{\infty}(-1)^{n} \mathbf{M}_{0 n}^{12} \alpha_{n}^{1}
\end{aligned}
$$

This term is also equal to $\sum \mathbf{M}_{0 n}^{21} \alpha_{n}^{1}$ by definition, and therefore $\mathbf{M}_{0 n}^{21}=(-1)^{n} \mathbf{M}_{0 n}^{12}$. The generic term $p>0$ yields:

$$
\begin{aligned}
E_{p}^{\prime} & +\frac{\mathrm{i}}{2} \sum_{m=0}^{p-1} E_{m}\left((-1)^{p-m} \beta_{p-m}+(-1)^{p+m} \beta_{p+m}\right)+\frac{\mathrm{i}}{2} E_{p}\left(2 \beta_{0}+\beta_{2 p}\right) \\
& +\frac{\mathrm{i}}{2} \sum_{m=p+1}^{\infty} E_{m}\left((-1)^{p-m} \beta_{m-p}+(-1)^{p+m} \beta_{p+m}\right) \\
=\sum_{n=0}^{\infty} & \frac{\alpha_{n}^{1}}{\epsilon_{n} H_{n}^{(1)}(k a)}\left[\left(J_{p}^{\prime}(k a)+\mathrm{i} \beta_{0} J_{p}(k a)+\frac{\mathrm{i}}{2} \beta_{2 p} J_{p}(k a)\right)\left((-1)^{n} H_{n+p}^{(1)}(k b)+H_{p-n}^{(1)}(k b)\right)\right. \\
& +\mathrm{i}(-1)^{p} \beta_{p}(-1)^{n} H_{n}^{(1)}(k b) J_{0}(k a) \\
& +\frac{\mathrm{i}}{2} \sum_{m=1}^{p-1}(\underbrace{(-1)^{p-m}}_{=(-1)^{p+m}} \beta_{p-m}+(-1)^{p+m} \beta_{p+m}) J_{m}(k a)\left((-1)^{n} H_{n+m}^{(1)}(k b)+H_{m-n}^{(1)}(k b)\right) \\
& +\frac{\mathrm{i}}{2} \sum_{m=p+1}^{\infty} \underbrace{(-1)^{m-p}}_{=(-1)^{m+p}} \beta_{m-p}+(-1)^{p+m} \beta_{p+m}) J_{m}(k a)\left((-1)^{n} H_{n+m}^{(1)}(k b)+H_{m-n}^{(1)}(k b)\right)]
\end{aligned}
$$




$$
\begin{aligned}
&=\sum_{n=0}^{\infty} \frac{(-1)^{p+n} \alpha_{n}^{1}}{\epsilon_{n} H_{n}^{(1)}(k a)}\left[(-1)^{p}\left(J_{p}^{\prime}(k a)+\mathrm{i} \beta_{0} J_{p}(k a)+\frac{\mathrm{i}}{2} \beta_{2 p} J_{p}(k a)\right)\left(H_{n+p}^{(1)}(k b)+(-1)^{n} H_{p-n}^{(1)}(k b)\right)\right. \\
&+\mathrm{i} \beta_{p} H_{n}^{(1)}(k b) J_{0}(k a) \\
&+\frac{\mathrm{i}}{2} \sum_{m=1}^{p-1}(-1)^{m}\left(\beta_{p-m}+\beta_{p+m}\right) J_{m}(k a)\left(H_{n+m}^{(1)}(k b)+(-1)^{n} H_{m-n}^{(1)}(k b)\right) \\
&\left.+\frac{\mathrm{i}}{2} \sum_{m=p+1}^{\infty}(-1)^{m}\left(\beta_{m-p}+\beta_{p+m}\right) J_{m}(k a)\left(H_{n+m}^{(1)}(k b)+(-1)^{n} H_{m-n}^{(1)}(k b)\right)\right] \\
&=\sum_{n=0}^{\infty}(-1)^{p+n} \mathbf{M}_{p n}^{12} \alpha_{n}^{1}=\sum_{n=0}^{\infty} \mathbf{M}_{p n}^{21} \alpha_{n}^{1}
\end{aligned}
$$

which yields the relationship: $(\forall p, n \in \mathbb{N}) \quad \mathbf{M}_{p n}^{21}=(-1)^{p+n} \mathbf{M}_{p n}^{12}$.

\section{Appendix A.2.4. Second line of the system of equations}

Putting everything together, the boundary condition on the image cylinder (A.5) becomes:

$$
\sum_{p=0}^{\infty} \cos \left(p \theta_{2}\right)\left[\sum_{n=0}^{\infty} \mathbf{M}_{p n}^{21} \alpha_{n}^{1}+\sum_{n=0}^{\infty} \mathbf{M}_{p n}^{22} \alpha_{n}^{2}\right]=\sum_{p=0}^{\infty} e_{p}^{2} \cos \left(p \theta_{2}\right)
$$

Again, by identification we have:

$$
(\forall p \in \mathbb{N}) \quad \sum_{n=0}^{\infty} \mathbf{M}_{p n}^{21} \alpha_{n}^{1}+\sum_{n=0}^{\infty} \mathbf{M}_{p n}^{22} \alpha_{n}^{2}=e_{p}^{2} \quad \Leftrightarrow \quad \mathbf{M}^{21} \boldsymbol{\alpha}^{1}+\mathbf{M}^{22} \boldsymbol{\alpha}^{2}=\mathbf{e}^{2}
$$

which is the second line of the system (11). 


\section{Appendix B. Derivatives with respect to the parameters}

In order to compute the sensitivity of the pressure field with respect to changes in the model parameters, we also need to compute the derivatives of the admittance function. For the resonant perforated panel model, this yields:

$$
\left\{\begin{array}{l}
\frac{\partial \gamma_{\mathrm{MPP}}}{\partial s}=-\mathrm{i} \gamma_{\mathrm{MPP}}^{2}\left[\frac{k l_{0}}{s^{2}}\left(\frac{1}{\Theta\left(x^{\prime}\right)}+\frac{16}{3 \pi} \frac{a_{0}}{l_{0}} \frac{\Psi(\xi)}{\Theta(x)}\right)-\frac{k a_{0}}{s} \frac{16}{3 \pi} \frac{\Psi^{\prime}(\xi)}{2 \xi \Theta(x)}\right] \\
\frac{\partial \gamma_{\mathrm{MPP}}}{\partial a_{0}}=\mathrm{i} \gamma_{\mathrm{MPP}}^{2} \frac{k l_{0}}{s}\left[-\frac{x^{\prime}}{a_{0}} \frac{\Theta^{\prime}\left(x^{\prime}\right)}{\Theta\left(x^{\prime}\right)^{2}}+\frac{16}{3 \pi} \frac{\Psi(\xi)}{l_{0}}\left(\frac{1}{\Theta\left(x^{\prime}\right)}-\frac{\Theta^{\prime}(x) x}{\Theta(x)^{2}}\right)\right] \\
\frac{\partial \gamma_{\mathrm{MPP}}}{\partial l_{0}}=\mathrm{i} \frac{k}{s} \gamma_{\mathrm{MPP}}^{2} \frac{1}{\Theta\left(x^{\prime}\right)} \\
\frac{\partial \gamma_{\mathrm{MPP}}}{\partial d}=\frac{\mathrm{i} k}{\sin ^{2}(k d)} \gamma_{\mathrm{MPP}}(f)^{2}
\end{array}\right.
$$

with the derivatives of the two functions $\Theta$ and $\Psi$ given by:

$$
\left\{\begin{array}{l}
\Psi^{\prime}(\xi)=\sum_{m=1}^{8} m u_{m} \xi^{m-1} \\
\Theta^{\prime}(w)=\frac{2}{w}\left(\frac{J_{2}(w \sqrt{\mathrm{i}})}{J_{0}(w \sqrt{\mathrm{i}})}-\frac{J_{1}(w \sqrt{\mathrm{i}})^{2}}{J_{0}(w \sqrt{\mathrm{i}})^{2}}\right)
\end{array}\right.
$$

For the rigid-backed Delany \& Bazley model, we have:

$$
\left\{\begin{aligned}
\frac{\partial \gamma_{\mathrm{DB}}}{\partial \sigma}= & -\gamma_{\mathrm{DB}}(f)^{2}\left[\operatorname{coth}(-\mathrm{i} \tilde{k} d)\left(0.75 \times 0.0511 \frac{1}{f}\left(\frac{\sigma}{f}\right)^{0.75-1}+\mathrm{i} 0.73 \times 0.0768 \frac{1}{f}\left(\frac{\sigma}{f}\right)^{0.73-1}\right)\right. \\
& \left.+\tilde{z}(f) \frac{\mathrm{i} k_{0} d}{\sinh ^{2}(-\mathrm{i} \tilde{k} d)} \frac{1}{f}\left(1+0.7 \times 0.0858\left(\frac{\sigma}{f}\right)^{0.7-1}+\mathrm{i} 0.59 \times 0.175\left(\frac{\sigma}{f}\right)^{0.59-1}\right)\right] \\
\frac{\partial \gamma_{\mathrm{DB}}}{\partial d}= & -\gamma_{\mathrm{DB}}(f)^{2} \frac{\mathrm{i} \tilde{k} \tilde{k}}{\sinh ^{2}(-\mathrm{i} \tilde{k} d)}
\end{aligned}\right.
$$

